\title{
LINEAR PROGRAMMING ESTIMATORS AND BOOTSTRAPPING FOR HEAVY TAILED PHENOMENA
}

\author{
Paul D. Feigin and Sidney I. Resnick \\ Technion, Israel Institute of Technology and Cornell University
}

March 6, 1995

\begin{abstract}
For autoregressive time series with positive innovations which either have heavy right or left tails, linear programming parameter estimates of the autoregressive coefficients have good rates of convergence. However, the asymptotic distribution of the estimators depends heavily on the distribution of the process and thus cannot be used for inference. A bootstrap procedure circumvents this difficulty. We verify the validity of the bootstrap and also give some general comments on the bootstrapping of heavy tailed phenomena.
\end{abstract}

\section{Introduction.}

Consider the stationary autoregressive process of order $p$, denoted by $\operatorname{AR}(p)$, with non-negative innovations $\left\{Z_{t}\right\}$, and with autoregressive coefficients $\phi_{1}, \ldots, \phi_{p}, \phi_{p} \neq 0$. These processes are defined by the following relation:

$$
X_{t}=\sum_{k=1}^{p} \phi_{k} X_{t-k}+Z_{t} ; t=0, \pm 1, \pm 2, \ldots
$$

where we assume that $\left\{Z_{t}\right\}$ is an independent and identically distributed (iid) sequence of random variables with left endpoint of their common distribution being 0 . We assume the order $p$ is known. Based on observation of $\left\{X_{1}, \ldots, X_{n}\right\}$ we are interested in estimating the parameters, and in determining the asymptotic properties of these estimators as $n \rightarrow \infty$.

The usual method of estimation would be to use the Yule-Walker estimators (see, for example, Brockwell and Davis (1991)), which would typically result in the estimators converging at the rate $n^{1 / 2}$. However, one can sometimes do better than the $n^{1 / 2}$ rate of convergence by exploiting the special nature of the innovations. This was the motivation behind the efforts, for example of Davis and McCormick (1989), Feigin and Resnick (1992,94), Andel (1989).

When either the left or the right tail of the $Z$ 's are regularly varying, Feigin and Resnick (1994) use the linear programming estimator (lp estimator) of the coefficients defined as follows. Suppose the true autoregressive parameter is $\phi(0)=\left(\phi_{1}(0), \ldots, \phi_{p}(0)\right)^{\prime}$. Our estimator is obtained by solving the linear program to get

$$
\hat{\phi}(n)=\arg \max _{\delta \in D_{n}} \delta^{\prime} \mathbf{1}
$$

\footnotetext{
Key words and phrases. Poisson processes, bootstrap, random measure, linear programming, autoregressive processes, parameter estimation, weak convergence, consistency, time series analysis.

Paul Feigin was partially supported by the United States-Israel Binational Science Foundation (BSF) Grant No. 92-00227/2. Sidney Resnick was supported by NSF Grant DMS-9400535 at Cornell University. His travel to Israel was supported by the Binational Science Foundation Grant .
} 
where $\mathbf{1}^{\prime}=(1, \ldots, 1)$ and where the feasible region $D_{n}$ is defined as

$$
D_{n}=\left\{\boldsymbol{\delta} \in \mathbb{R}^{p}: X_{t}-\sum_{i=1}^{p} \delta_{i} X_{t-i} \geq 0 ; t=p+1, \ldots, n\right\} .
$$

The fundamental properties of this estimator were discussed in Feigin and Resnick (1994) and some applications to testing for independence were given in Feigin, Resnick and Starica (1994). Under either a left or right tail regular variation condition, there exist $r(n) \rightarrow \infty$ such that $r(n)(\hat{\phi}(n)-\phi(0))$ has a limit distribution. While the rates of convergence for the lp estimator can be excellent, it possesses the unfortunate characteristic that the limiting distribution for $\hat{\phi}(n)$ depends on the unknown distribution of the time series. For inference purposes, this characteristic is a serious flaw. However, we propose to overcome this problem via the use of the bootstrap paradigm.

Here is a description of our bootstrap procedure; it is a generalization of one used by Datta and McCormick (1995). The steps of the procedure are as follows:

(1) Assume we observe $X_{1}, \ldots, X_{n}$ where $\left\{X_{t}\right\}$ is a $p$ th order stationary autoregressive process as in (1.1) satisfying tail conditions guaranteeing asymptotic distributions for the lp estimator.

(2) We estimate the autoregressive coefficients using the lp estimators and obtain a vector of estimates $\hat{\phi}(n)$.

(3) We then use these estimates $\hat{\phi}(n)$ to estimate the residuals

$$
\hat{Z}_{t}(n)=X_{t}-\sum_{i=1}^{p} \hat{\phi}_{i}(n) X_{t-i} ; \quad t=p+1, \ldots, n
$$

For the lp estimator $\hat{\phi}(n), p$ of these residuals will equal 0 .

(4) We form the empirical measure generated by the non-zero estimated residuals

$$
\hat{F}_{n}^{r e s i d}=\frac{1}{n-2 p} \sum_{i=p+1}^{n} 1_{\left[\hat{Z}_{i}(n)>0\right]} \epsilon_{\hat{Z}_{i}(n)} .
$$

(5) Resample: Draw an iid sample $\left\{Z_{t}^{*}(n) ; t=1, \ldots, m\right\}$ from the distribution $\hat{F}_{n}^{\text {resid }}$ where, asymptotically, $m=m(n) \rightarrow \infty$ but $m / n \rightarrow 0$ as $n \rightarrow \infty$.

(6) Construct a bootstrap time series $\left\{X_{t}^{*}(n) ; t=1, \ldots, m\right\}$ by setting $X_{0}^{*}(n)=\cdots=X_{-p+1}^{*}(n)=0$ and then using the recursion

$$
X_{t}^{*}(n)=\sum_{i=1}^{p} \hat{\phi}_{i}(n) X_{t-i}^{*}(n)+Z_{t}^{*}(n) .
$$

(7) Define

$$
\hat{\phi}^{*}(n)=\arg \max \left\{\sum_{i=1}^{p} \delta_{i}: X_{t}^{*}(n)-\sum_{i=1}^{p} \delta_{i} X_{t-i}^{*}(n) \geq 0 ; t=p+1, \ldots, m\right\} .
$$

(8) The ideal bootstrap distribution is the sampling distribution, conditional on $\hat{F}_{n}^{\text {resid }}$, of

$$
r(m)\left(\hat{\phi}^{*}(n)-\hat{\phi}(n)\right) .
$$

This cannot be computed exactly so in practice Monte Carlo is necessary. Also, a modification of this method is necessary to account for the fact that $r(m)$ is unknown. 
One notes in the described procedure that the bootstrap sample size is of smaller order than the sample size of the observed data. In connection with bootstrapping extremes and heavy tailed phenomena, many authors have noticed that if the original sample is of size $n$, in order for the bootstrap asymptotics to work as desired the bootstrap sample should be of size $m$ where $m$ is a function of $n$ and $m / n \rightarrow 0$ as $n \rightarrow \infty$. See for example Athreya (1987), Gine and Zinn (1989), Hall (1990), Kinateder (1992), Knight (1989), Lepage (1992), and Deheuvels, Mason and Shorack (1993).

Section 2 gives some perspective on this necessity to reduce the bootstrap sample size to something of smaller order than the observed sample size through a discussion of the behavior of random measures determined by the bootstrap sample. Section 3 gives a random measure version of a theorem of Karamata (see for example, Feller (1971); Bingham, Goldie and Teugels (1987); de Haan (1970); Geluk and de Haan (1987) or Resnick (1987)) which discusses the regular variation behavior of integrals of regularly varying functions. This Karamata generalization is needed in Section 5 where we show the asymptotic validity of the bootstrap for the lp estimator. In Section 4 are triangular array point process limit theorems which provide the theoretical underpinning for Section 5.

We now give a precise statement of conditions and results proven in previous papers that will be needed here. We need conditions which specify the model and guarantee stationarity. In order to obtain a limit distribution for our estimators, we impose regular variation and moment conditions on the distribution of the innovation sequence. We recall that a function $U:[0, \infty) \mapsto[0, \infty)$ is regularly varying with exponent $\rho \in \mathbb{R}(U \in \mathbb{R V}(\rho))$ at infinity if

$$
\lim _{t \rightarrow \infty} \frac{U(t x)}{U(t)}=x^{\rho}, \quad x>0 .
$$

A parallel definition holds for regular variation at 0 .

We now state precise conditions under which our results will hold:

(1) Condition $\mathbf{M}$ (model specification): The process $\left\{X_{t}: t=0, \pm 1, \pm 2, \ldots\right\}$ satisfies the equations

$$
X_{t}=\sum_{i=1}^{p} \phi_{i} X_{t-i}+Z_{t}, \quad t=0, \pm 1, \pm 2, \ldots
$$

where $\left\{Z_{t}\right\}$ is an independent and identically distributed sequence of positive random variables with common distribution function $F$.

(2) Condition $\mathbf{S}$ (stationarity): The coefficients $\phi_{1}, \ldots, \phi_{p}$ satisfy the stationarity condition that the autoregressive polynomial $\Phi(z) \equiv 1-\sum_{1}^{p} \phi_{i} z^{i}$ has no roots in the unit disk $\{z:|z| \leq 1\}$. Note that by continuity of $\Phi(x)$ for $x$ real, we also get $\Phi(1)>0$; i.e.

$$
\sum_{i=1}^{p} \phi_{i}<1
$$

(3) Condition $\mathbf{L}$ (left tail): The distribution $F$ of the innovations $Z_{t}$ satisfies, for some $\alpha>0$ :

1. $\lim _{s \downarrow 0} \frac{F(s x)}{F(s)}=x^{\alpha}$ for all $x>0$;

2. $E\left(Z_{t}^{\beta}\right)=\int_{0}^{\infty} u^{\beta} F(d u)<\infty$ for some $\beta>\alpha$.

(4) Condition $\mathbf{R}$ (right tail): The distribution $F$ of the innovations $Z_{t}$ satisfies, for some $\alpha>0$ :

1. $\lim _{s \rightarrow \infty} \frac{1-F(s x)}{1-F(s)}=x^{-\alpha}$ for all $x>0$;

2. $E\left(Z_{t}^{-\beta}\right)=\int_{0}^{\infty} u^{-\beta} F(d u)<\infty$ for some $\beta>\alpha$. 
Define two quantile functions

$$
a(n)=F^{\leftarrow}\left(\frac{1}{n}\right), \quad b(n)=F^{\leftarrow}\left(1-\frac{1}{n}\right) .
$$

Our results have as hypotheses M, S, and either L or R. For additional remarks concerning these conditions see Feigin and Resnick (1992). However, note that Condition L is rather mild. It is satisfied if a density $f$ of $F$ exists which is continuous at 0 and with $f(0)>0$. In this case $\alpha=1$. Other common cases where Condition L holds are the Weibull distributions of the form $F(x)=1-\exp \left\{-x^{\alpha}\right\}$ where $F(x) \sim x^{\alpha}$, as $x \downarrow 0$ and the gamma densities $f(x)=c e^{-x} x^{r-1}, r>0, x>0$ so that $f(x) \sim c x^{r-1}$ as $x \downarrow 0$ and therefore the associated Gamma distribution function satisfies $F(x) \sim c r^{-1} x^{r}$, as $x \downarrow 0$. Examples of distributions satisfying condition $\mathrm{R}$ include positive stable densities and the Pareto density.

Assuming Conditions $\mathrm{M}, \mathrm{S}$, and either $\mathrm{L}$ or $\mathrm{R}$ hold, if the true model is $\mathrm{AR}(p)$ given by (1.3) with true parameter vector $\phi(0)$, then for $r(n)$ suitably chosen to be either $a(n)^{-1}$ or $b(n)$ there is a limit distribution

$$
r(n)(\hat{\phi}(n)-\phi(0)) \Rightarrow \mathbf{L}
$$

for some limiting random variable $\mathbf{L}$. The limit distribution corresponding to $\mathbf{L}$ is quite complicated in general and depends on the unknown $\alpha$ and the finite dimensional distributions of the process $\left\{X_{t}\right\}$. The precise statement is given next and is culled from Feigin and Resnick (1994) and Feigin, Resnick and Starica (1994).

Theorem 1.1. Suppose conditions $M$ and $S$ hold and let $\hat{\phi}(n)$ be the linear programming estimator based on $X_{1}, \ldots, X_{n}$ given in (1.5) and (1.6). Let $\left\{E_{j}, j \geq 1\right\}$ be iid unit exponential random variables and define

$$
\Gamma_{k}=E_{1}+\cdots+E_{k}, \quad k \geq 1,
$$

so that $\left\{\Gamma_{k}\right\}$ are the points of a homogeneous Poisson process.

(a) If Condition $L$ holds, then

$$
a(n)^{-1}(\hat{\phi}(n)-\phi(0)) \Rightarrow \mathbf{L}
$$

where $\mathbf{L}$ is non-degenerate and

$$
\mathbf{L} \stackrel{d}{=} \arg \max _{\delta \in \Lambda} \boldsymbol{\delta}^{\prime} \mathbf{1}
$$

where

$$
\Lambda=\left\{\boldsymbol{\delta} \in R^{p}: \boldsymbol{\delta}^{\prime} \mathbf{1} \geq-1, \boldsymbol{\delta}^{\prime} \mathbf{u}_{k} \leq 1, k \geq 1\right\} .
$$

The points $\left\{\mathbf{u}_{k}\right\}$ are specified as follows. Let $\left\{\mathbf{Y}_{j} ; j \geq 1\right\}$ be iid $\mathbb{R}^{p}$ valued random vectors with

$$
\mathbf{Y}_{1} \stackrel{d}{=}\left(X_{p}, \ldots, X_{1}\right)
$$

and suppose $\left\{\mathbf{Y}_{j}, j \geq 1\right\}$ are independent of $\left\{\Gamma_{k}\right\}$. Then

$$
\mathbf{u}_{k}=\Gamma_{k}^{-1 / \alpha} \mathbf{Y}_{k} .
$$

(b) Suppose Condition $R$ holds. Define for $|z| \leq 1$

$$
C(z)=\sum_{j=0}^{\infty} c_{j} z^{j}=\frac{1}{\Phi(z)}
$$


and let $\mathbf{c}_{l}=\left(c_{l}, c_{l-1}, \ldots, c_{l-p+1}\right)^{\prime}$. Then

$$
b(n)(\hat{\phi}(n)-\phi(0))=O_{p}(1)
$$

so the rate of convergence of $\hat{\phi}(n)$ to $\phi(0)$ is $b(n)$. Furthermore, if for any $p-1$ distinct indices $\left\{l_{1}, \ldots, l_{p-1}\right\}$, for which the set of $p$ vectors

$$
\left\{1, \mathbf{c}_{l_{1}}, \ldots, \mathbf{c}_{l_{p-1}}\right\}
$$

does not contain the zero vector, the set is also linearly independent, then

$$
b(n)(\hat{\phi}(n)-\phi(0)) \Rightarrow \mathbf{L}
$$

where $\mathbf{L}$ is non-degenerate,

$$
\mathbf{L} \stackrel{d}{=} \arg \max _{\delta \in \Lambda} \delta^{\prime} \mathbf{1}
$$

and

$$
\Lambda=\left\{\boldsymbol{\delta} \in R^{p}: \boldsymbol{\delta}^{\prime} \mathbf{1} \geq-1, \boldsymbol{\delta}^{\prime} \mathbf{v}_{l} \leq 1, l \geq 0\right\} .
$$

The points $\left\{\mathbf{v}_{l}\right\}$ are specified as follows. Let $\left\{Y_{k l} ; k \geq 1, l \geq 0\right\}$ be a doubly infinite array of iid random variables which is independent of $\left\{\Gamma_{k}\right\}$ with the distribution $F$. Then

$$
\mathbf{v}_{l}=\left(\bigvee_{k=1}^{\infty} \Gamma_{k}^{-1 / \alpha} Y_{k l}^{-1}\right) \mathbf{c}_{l}=V_{l} \mathbf{c}_{l} ; \quad l=0,1,2, \ldots
$$

\section{Random measures and bootstrapping heavy tailed phenomena.}

We now proceed to explain the nature of the bootstrap approximation to the asymptotic distribution of the estimator sequence $\{\hat{\phi}(n)\}$ described above. To do so we introduce some notation for random measures since the asymptotic theory described above is based on weak convergence of random measures. We will learn why subsampling - choosing a bootstrap sample of size $m=o(n)$ - is required for the bootstrap asymptotics of extremes and heavy tailed phenomena.

Let $M_{+}(E)$ denote the set of positive Radon measures on a nice locally compact space $E . M_{+}(E)$ is metrized by the vague metric (cf. Kallenberg, 1983; Resnick, 1987) which we will denote by $\rho$ in the sequel. We denote weak convergence of random elements or probability measures by " $\Rightarrow$ " while " $\rightarrow$ " denotes vague convergence of measures in $M_{+}(E)$. For $x \in E$ and $A \subset E$ define

$$
\epsilon_{x}(A)= \begin{cases}1, & \text { if } x \in A, \\ 0, & \text { otherwise. }\end{cases}
$$

A Radon point measure with points in $E$ is denoted $\sum_{i} \epsilon_{x_{i}}$. The collection of all such point measures is $M_{p}(E)$. We denote by $\operatorname{PRM}(\nu)$ the Poisson Random Measure in $M_{p}(E)$ with mean measure $\nu$ on $E$.

The asymptotic theory is based on the convergence of random measures generated from $n$ iid observations $\left\{Z_{1}, \ldots, Z_{n}\right\}$ drawn from a given distribution $F$ on $E$. Let $m=m(n)$ in the following. Denote by

$$
\begin{aligned}
& \xi_{n}(F)=\frac{1}{n} \sum_{t=1}^{n} \epsilon_{Z_{t}} \\
& \mu_{n}(F)=\sum_{t=1}^{n} \epsilon_{Z_{t} / q(n)}=n \xi_{n}(F)(q(n) \cdot) \\
& \nu_{n}(F)=\frac{m}{n} \sum_{t=1}^{n} \epsilon_{Z_{t} / q(m)}=m \xi_{n}(F)(q(m) \cdot) .
\end{aligned}
$$


Remarks. The random measure $\xi_{n}(F)$ is the empirical distribution based on a sample of size $n$ from $F$. The mean measures of the above random measures are given by

$$
E\left[\xi_{n}(F)\right]=F, \quad E\left[\mu_{n}(F)\right]=n F(q(n) \cdot), \quad E\left[\nu_{n}(F)\right]=m F(q(m) \cdot) .
$$

The distribution of the random measures are determined by $F$ and $n$ and for $\mu_{n}(F)$ and $\nu_{n}(F)$ by the choice of sequences $q(n)$ and $m(n)$. For $E=[0, \infty)$ and $q(n)=a(n), F \in R V(\alpha)$ at 0 is equivalent to

$$
E \mu_{n}(F)(\cdot)=n F(q(n) \cdot) \stackrel{v}{\rightarrow} \nu(\cdot) \text { with } \nu([0, x])=x^{\alpha} .
$$

Likewise, for $E=(0, \infty]$ and $q(n)=b(n), 1-F \in R V(-\alpha)$ at $\infty$ is equivalent to

$$
E \mu_{n}(F)(\cdot)=n F(q(n) \cdot) \stackrel{v}{\rightarrow} \nu(\cdot) \text { with } \nu((x, \infty])=x^{-\alpha} .
$$

A bootstrap sample can be thought of as an iid sample from the empirical distribution $\xi_{n}(F)$. If that sample was of size $n$ then the distribution of the appropriate bootstrapped point process would be the conditional distribution of $\mu_{n}\left(\xi_{n}(F)\right)$ given $\xi_{n}(F)$. The bootstrap distribution will, asymptotically, be close to the desired asymptotic distribution $\operatorname{PRM}(\nu)$ if

$$
P\left[\mu_{n}\left(\xi_{n}(F)\right) \in \cdot \mid \xi_{n}(F)\right]
$$

would converge in an appropriate sense to $\operatorname{PRM}(\nu)$. However, this does not happen as we explain below.

What does work is to consider the conditional distribution of $\mu_{m}\left(\xi_{n}(F)\right)$ given $\xi_{n}(F)$ where $m=m(n) \rightarrow$ $\infty, m(n)=\mathrm{o}(n)$. The following proposition provides the underlying result in this direction. Let

$$
\pi_{n}(F)=\sum_{t=1}^{n} \epsilon_{Z_{t}}=n \xi_{n}(F)
$$

Proposition 2.1. Let $\left\{F_{n}\right\}$ be a sequence of distributions on $E$.

(a) $\pi_{n}\left(F_{n}\right) \Rightarrow \operatorname{PRM}(\nu)$ iff $E\left[\pi_{n}\left(F_{n}\right)\right]=n F_{n} \stackrel{v}{\rightarrow} \nu$.

(b) Suppose $m(n) \rightarrow \infty, m=o(n)$. Then

$\frac{m}{n} \pi_{n}\left(F_{n}\right)=m \xi_{n}\left(F_{n}\right) \stackrel{P}{\rightarrow} \nu$ in $\left(M_{+}(E), \rho\right)$ iff $E\left[\frac{m}{n} \pi_{n}\left(F_{n}\right)\right]=m F_{n} \stackrel{v}{\rightarrow} \nu$.

Proof. Part (a) is Proposition 3.21, page 154 and part (b) is Proposition 3.57, page 161 in Resnick (1987).

The application to the RV cases is immediate. Let $F_{n}(\cdot)=F(q(n) \cdot)$ so that if $Z_{t}(n)$ has distribution $F_{n}$, then $Z_{t}(n) \stackrel{d}{=} Z_{t} / q(n)$, where $Z_{t}$ has distribution $F$. Then we have the following equivalences:

(1) $n F(q(n) \cdot) \stackrel{v}{\rightarrow} \nu$ in $M_{+}(E)$;

(2) $\mu_{n}(F) \Rightarrow \operatorname{PRM}(\nu)$ in $M_{p}(E)$;

(3) $\nu_{n}(F) \stackrel{P}{\rightarrow} \nu$ in $\left(M_{+}(E), \rho\right)$.

Note that (3) is equivalent to (1) by setting $F_{n}(\cdot)=F(q(m(n)) \cdot)$ in Proposition 2.1(b).

Now we apply the foregoing to the subsampled bootstrap random measure

$$
\mu_{m}\left(\xi_{n}(F)\right)=\sum_{t=1}^{m} \epsilon_{Z_{t}^{*} / q(m)}, Z_{t}^{*} \text { iid from } \xi_{n}(F) .
$$

Denote by $\mathcal{P} \mathcal{M}\left(M_{p}(E)\right)$ the (separable, metric) space of probability measures on $M_{p}(E)$. 
Proposition 2.2. Suppose $m(n)=o(n), m(n) \rightarrow \infty$ as $n \rightarrow \infty$ and $n F(q(n) \cdot) \stackrel{v}{\rightarrow} \nu$ in $M_{+}(E)$. Then, as $n \rightarrow \infty$,

$$
P\left[\mu_{m}\left(\xi_{n}(F)\right) \in \cdot \mid \xi_{n}(F)\right] \stackrel{P}{\rightarrow} P[P R M(\nu) \in \cdot]
$$

in $\mathcal{P} \mathcal{M}\left(M_{+}(E)\right)$.

Proof. We have

$$
E\left[\mu_{m}\left(\xi_{n}(F)\right) \mid \xi_{n}(F)\right]=\nu_{n}(F) \stackrel{P}{\rightarrow} \nu
$$

from (3) above. The required result then follows from the following lemma.

Lemma 2.3. IF $\left\{G_{n}\right\}$ is a sequence of random probability measures and

$$
E\left[\mu_{m}\left(G_{n}\right) \mid G_{n}\right] \stackrel{P}{\rightarrow} \nu \text { in }\left(M_{+}(E), \rho\right)
$$

then

$$
P\left[\mu_{m}\left(G_{n}\right) \in \cdot \mid G_{n}\right] \stackrel{P}{\rightarrow} P[P R M(\nu) \in \cdot] \text { in } \mathcal{P} \mathcal{M}\left(M_{p}(E)\right) ;
$$

with the topology of weak convergence.

Proof. For any subsequence $\left\{n^{\prime \prime}\right\}$ of $\{n\}$ choose a further subsequence $\left\{n^{\prime}\right\}$ along which

$$
E\left[\mu_{m\left(n^{\prime}\right)}\left(G_{n^{\prime}}\right) \mid G_{n^{\prime}}\right] \rightarrow \nu \text { a.s. . }
$$

Then by Proposition 2.1(a),

$$
P\left[\mu_{m\left(n^{\prime}\right)}\left(G_{n^{\prime}}\right) \in \cdot \mid G_{n^{\prime}}\right] \rightarrow P[\operatorname{PRM}(\nu) \in \cdot] \text { a.s. . }
$$

By the usual subsequence argument for convergence in probability we conclude that

$$
P\left[\mu_{m}\left(G_{n}\right) \in \cdot \mid G_{n}\right] \stackrel{P}{\rightarrow} P[\operatorname{PRM}(\nu) \in \cdot] \text { in } \mathcal{P} \mathcal{M}\left(M_{p}(E)\right)
$$

as required.

Proposition 2.2 provides us with the motivation to subsample - only then will the bootstrap distribution of the point process approximate the true asymptotic distribution of the original point process.

An interesting question, thought not central to this paper, is what happens in the limit to the full-sample bootstrap random point process $\mu_{n}\left(\xi_{n}(F)\right)$. The answer can be given as follows.

Proposition 2.4. Suppose $n F(q(n) \cdot) \stackrel{v}{\rightarrow} \nu$ with $\nu([x, \infty))=x^{-\alpha}, q(n)=b(n)$ and $E=(0, \infty]$ in the right tail case and $\nu([0, x])=x^{\alpha}, q(n)=a(n)$ and $E=[0, \infty)$ in the left tail case. Then in $\mathcal{P} \mathcal{M}\left(M_{p}(E)\right)$

$$
P\left[\mu_{n}\left(\xi_{n}(F)\right) \in \cdot \mid \xi_{n}(F)\right] \Rightarrow P\left[\sum_{k=1}^{\infty} P O_{k} \epsilon_{j_{k}} \in \cdot \mid\left\{j_{k}\right\}\right]
$$

where $\left\{j_{k}\right\}$ are the points of a $P R M(\nu)$ process and $\left\{P O_{k}\right\}$ are iid Poisson mean 1 random variables independent of $\left\{j_{k}\right\}$.

Proof. We provide an outline of the proof. For the space of sequences $\left\{y_{i}\right\} \subset E$, either finite or countable, define a metric $d$ as follows:

$$
d\left(\left\{y_{i}\right\},\left\{z_{i}\right\}\right)=\rho\left(\sum \epsilon_{y_{i}}, \sum \epsilon_{z_{i}}\right)
$$


so that we are really dealing with equivalence classes of sequences (having the same elements but possibly ordered differently) which give rise to Radon point measures on $E$ - call this metric space $S Q(E)$. Given a sequence, define a random point process $\eta_{n}\left(\left\{y_{i}\right\}\right)$ by

$$
\eta_{n}\left(\left\{y_{i}\right\}\right)=\sum_{i=1}^{n} P_{i}(n) \epsilon_{y_{i}}
$$

where $\left(P_{1}(n), \ldots, P_{n}(n)\right)$ is a multinomial random vector with $n$ trials and equal probabilities $1 / n$. If $\left\{y_{i}\right\}$ is finite, then the sum in (2.1) is up to $\min (n, C)$ where $C=\#\left\{y_{i}\right\}$ is the cardinality of the sequence.

Now define a sequence of mappings $h_{n}$ from $S Q(E)$ to $\mathcal{P} \mathcal{M}\left(M_{p}(E)\right)$ given by

$$
h_{n}\left(\left\{y_{i}\right\}\right)=P\left[\eta_{n}\left(\left\{y_{i}\right\}\right) \in \cdot\right] .
$$

We now show that $h_{n} \rightarrow h$ where

$$
h\left(\left\{y_{i}\right\}\right)=P\left[\eta\left(\left\{y_{i}\right\}\right) \in \cdot\right] ; \quad \eta\left(\left\{y_{i}\right\}\right)=\sum_{k=1}^{C} P O_{k} \epsilon_{y_{k}} ;
$$

and $\left\{P O_{k}\right\}$ is a sequence of iid Poisson mean 1 random variables. The latter is achieved by showing that for all $f \in C_{K}^{+}(E)$,

$$
E\left[e^{-\int f d \eta_{n}}\right]=\left(1-\frac{\sum\left(1-e^{-f\left(y_{i}\right)}\right)}{n}\right)^{n} \rightarrow \exp \left\{-\sum\left(1-e^{-f\left(y_{i}\right)}\right)\right\}=E\left[e^{-\int f d \eta}\right] .
$$

The last equality can be derived as follows:

$$
\begin{aligned}
E\left(e^{-\int f(x) \eta(d x)}\right) & =E\left(e^{-\sum_{k} P O_{k} f\left(y_{k}\right)}\right)=\prod_{k=1}^{\infty} \exp \left\{e^{-f\left(y_{k}\right)}-1\right\} \\
& =\exp \left\{-\int\left(1-e^{-f(x)}\right) \sum_{k=1}^{\infty} \epsilon_{y_{k}}(d x)\right\}
\end{aligned}
$$

A simple extension of the above argument will also confirm that for a sequence $\mathbf{y}(n)=\left\{y_{i}(n)\right\}$ of sequences in $S Q(E)$ satisfying $d(\mathbf{y}(n), \mathbf{y}) \rightarrow 0$, whereupon

$$
\sum_{i=1}^{\infty} \epsilon_{y_{i}(n)} \stackrel{v}{\rightarrow} \sum_{i=1}^{\infty} \epsilon_{y_{i}},
$$

then also $h_{n}(\mathbf{y}(n)) \rightarrow h(\mathbf{y})$ in $\mathcal{P} \mathcal{M}\left(M_{p}(E)\right)$.

The latter continuous convergence result is enough to apply the so-called second continuity theoremBillingsley(1968, page 34). In other words, since

$$
\left\{Z_{t} / q(n) ; t=1, \ldots, n\right\} \Rightarrow\left\{j_{k}\right\}
$$

with respect to the metric $d$ on $S Q(E)$ we can conclude that

$$
h_{n}\left(\left\{Z_{t} / q(n) ; t=1, \ldots, n\right\}\right) \Rightarrow h\left(\left\{j_{k}\right\}\right)
$$

in the space $\mathcal{P} \mathcal{M}\left(M_{p}(E)\right)$. The latter is just a restatement of the assertion of the Proposition. 
We now proceed to explain the main ideas behind our results for bootstrapping for the autoregressive process (1.1). Since we will base the bootstrap on the observed residuals we need to use a more complicated random measure construction. Thus, given $F$ and $\phi \in \mathbb{R}^{p}$ we denote by $\lambda_{n}(F ; \phi)$ a random point measure as follows:

$$
\lambda_{n}(F ; \phi)= \begin{cases}\sum_{t=1}^{n} \epsilon_{\left(Z_{t} / a(n), \boldsymbol{X}_{t-1}\right),} & \text { in } M_{+}\left([0, \infty) \times((-\infty, \infty) \backslash\{0\})^{p}\right) \text { in case (L) } \\ \sum_{t=1}^{n} \epsilon_{\left(Z_{t}, b(n)^{-1} \boldsymbol{X}_{t-1}\right)}, & \text { in } M_{+}\left((0, \infty) \times\left([-\infty, \infty]^{p} \backslash\{0\}\right)\right) \text { in case (R) }\end{cases}
$$

where $Z_{1}, \ldots, Z_{n}$ are iid $F ; Z_{j}=0, j \leq 0 ; X_{t}=0, t \leq 0$;

$$
\begin{aligned}
X_{t} & =Z_{t}+\sum_{j=1}^{p} \phi_{j} X_{t-j}, t \geq 1 \\
& =\sum_{j=0}^{\infty} c_{j} Z_{t-j}, t \geq 1 ;
\end{aligned}
$$

$\mathbf{X}_{t}=\left(X_{t}, X_{t-1}, \ldots, X_{t-p+1}\right)^{\prime}, \mathbf{c}_{j}=\left(c_{j}, \ldots, c_{j-p+1}\right)^{\prime}-$ the $c_{j}$ are defined in (1.3) via $C(z)=1 / \Phi(z)$; so that from (2.4) we can also write

$$
\mathbf{X}_{t}=\sum_{j=0}^{\infty} \mathbf{c}_{j} Z_{t-j}
$$

A key step in the proof of Theorem 1.1 is to show that

$$
\begin{gathered}
\lambda_{n}(F ; \phi) \Rightarrow \lambda_{\infty} \\
T \lambda_{n}(F ; \phi) \Rightarrow T \lambda_{\infty}
\end{gathered}
$$

where $T: M_{p}(E) \mapsto M_{p}\left(E^{\prime}\right)$ is defined by

$$
T\left(\sum_{t=1}^{\infty} \epsilon_{\left(x_{t}, \mathbf{v}_{t}\right)}\right)=\sum_{t=1}^{\infty} \epsilon_{x_{t}^{-1} \mathbf{v}_{t}} .
$$

In the above, $\lambda_{\infty}$ is of course different in the left (L) and right $(\mathrm{R})$ tail cases. Note that $T$ is not a continuous map for the cases $E=[0, \infty) \times((-\infty, \infty) \backslash\{0\})^{p}$ or $E=(0, \infty) \times[-\infty, \infty]^{p} \backslash\{\mathbf{0}\}$ with $E^{\prime}=[-\infty, \infty]^{p} \backslash\{\mathbf{0}\}$. Thus proving (2.7) from (2.6) involves extra conditions needed to make a truncation argument work.

The bootstrap will succeed if, for suitable $m=m(n)=\mathrm{o}(n)$,

$$
T \lambda_{m}\left(\hat{F}_{n}^{r e s i d} ; \hat{\phi}(n)\right) \stackrel{P}{\rightarrow} T \lambda_{\infty} \text { in } \mathcal{P} \mathcal{M}\left(M_{p}\left(E^{\prime}\right)\right)
$$

In order to achieve such a result we first seek triangular array extensions of (2.6) and (2.7) above. They provide conditions (COND) on $\left\{F_{n}\right\}$ and $\{\phi(n)\}$ for which

$$
T \lambda_{m}\left(F_{n} ; \phi(n)\right) \Rightarrow T \lambda_{\infty} .
$$

The program will be complete if we can show under suitable conditions, at least along a subsequence $\left\{n^{\prime}\right\}$, that $\left\{\hat{F}_{n^{\prime}}^{\text {resid }}\right\}$ and $\left\{\hat{\phi}\left(n^{\prime}\right)\right\}$ satisfy COND almost surely. Then, just as in Lemma 2.3, we can conclude that (2.9) holds and the bootstrap is successful asymptotically. 
We now collect some basic relationships that will be used throughout the proofs. They relate to showing that the estimated residuals $\left\{\hat{Z}_{t}(n)\right\}$ are close to the true residuals $\left\{Z_{t}\right\}$, and to bounding the moments of $\left\|\mathbf{X}_{t}\right\|$. For the estimated residuals $\hat{Z}_{t}(n)$ we have

$$
\begin{aligned}
\hat{Z}_{t}(n) & =Z_{t}-(\hat{\phi}(n)-\phi(0))^{\prime} \mathbf{X}_{t-1} \\
& =Z_{t}\left(1-r(n)(\hat{\phi}(n)-\phi(0))^{\prime}\left(r(n)^{-1} Z_{t}^{-1} \mathbf{X}_{t-1}\right)\right. \\
& =Z_{t}\left(1-\hat{\delta}(n)^{\prime} \mathbf{u}_{t}(n)\right)
\end{aligned}
$$

where

$$
\left\{\mathbf{u}_{t}(n)=r(n)^{-1} Z_{t}^{-1} \mathbf{X}_{t-1} ; t=1, \ldots, n\right\}
$$

are the points of $T \lambda_{n}(F ; \phi(0))$, and

$$
\hat{\boldsymbol{\delta}}(n)=\arg \max \left\{\boldsymbol{\delta} \mathbf{1}: \boldsymbol{\delta}^{\prime} \mathbf{u}_{t}(n) \leq 1, t=1, \ldots, n\right\}=r(n)(\hat{\boldsymbol{\phi}}(n)-\boldsymbol{\phi}(0)) .
$$

Letting $\Delta_{t}(n)=\hat{Z}_{t}(n)-Z_{t}$ we learn from the above that

$$
\Delta_{t}(n)=-Z_{t} \hat{\delta}(n)^{\prime} \mathbf{u}_{t}(n)=-(\hat{\phi}(n)-\phi(0))^{\prime} \mathbf{X}_{t-1}
$$

so that

$$
\left|\Delta_{t}(n)\right| \leq\|\hat{\phi}(n)-\phi(0)\|\left\|\mathbf{X}_{t-1}\right\|=R_{t}(n)
$$

and

$$
\hat{Z}_{t}(n) \geq \kappa Z_{t} \text { on } \hat{\delta}(n)^{\prime} \mathbf{u}_{t}(n) \leq 1-\kappa .
$$

From

$$
\mathbf{X}_{t}=\sum_{j=0}^{\infty} \mathbf{c}_{j} Z_{t-j}
$$

we have

$$
\begin{gathered}
\left\|\mathbf{X}_{t}\right\| \leq \sum_{j=0}^{\infty}\left\|\mathbf{c}_{j}\right\| Z_{t-j} ; \\
E\left\|\mathbf{X}_{t}\right\|^{\beta} \leq \sum_{j=0}^{\infty}\left\|\mathbf{c}_{j}\right\|^{\beta} E\left(Z_{1}\right)^{\beta} ; \text { for } \beta \leq 1
\end{gathered}
$$

and

$$
E\left\|\mathbf{X}_{t}\right\|^{\beta} \leq \sum_{j=0}^{\infty}\left\|\mathbf{c}_{j}\right\|^{s \beta} E\left(Z_{1}^{\beta}\right)\left(\sum_{j=0}^{\infty}\left\|\mathbf{c}_{j}\right\|^{(1-s) \beta /(\beta-1)}\right)^{\beta-1} ; \text { for } \beta>1 \text { and } 0<s<1
$$

using the Hölder inequality. 


\section{A stochastic version of Karamata's theorem.}

Karamata's theorem is a basic result in the theory of regularly varying functions which describes the regular variation properties of integrals of regularly varying functions. See, for instance Feller (1971); Bingham, Goldie and Teugels (1987); de Haan (1970); Geluk and de Haan (1987) or Resnick (1987).

In this section we present some stochastic versions of this theorem. Instead of integrating tail probabilities, we will integrate tail empirical measures and obtain limits in probability as if we had integrated the tail probabilities. The tail empirical measures we consider are those generated by the original $\left\{Z_{t}, 1 \leq t \leq n\right\}$ as well as those generated by the estimated residuals of the autoregression $\left\{\hat{Z}_{t}(n) ; p+1 \leq t \leq n, \hat{Z}_{t}(n)>0\right\}$.

We start with the simpler case first. We let $C_{0}[0, \infty)$ denote the space of continuous functions on $[0, \infty)$, with value 0 at 0 , and topologized by local uniform convergence.

Proposition 3.1. Suppose $\left\{Z_{t}, t \geq 1\right\}$ are iid with common distribution $F$ and suppose $P\left[Z_{t} \geq 0\right]=1$. Write $\nu_{n}(\cdot)$ for $\nu_{n}(F)(\cdot)$.

(a) Suppose Condition $R$ holds so that $1-F \in R V(-\alpha)$ at $\infty$. Then in $C_{0}[0, \infty)$ we have for any $\beta>\alpha$

$$
\int_{0}^{x} \nu_{n}(u, \infty] u^{\beta-1} d u=\int_{0}^{x} \frac{m}{n} \sum_{t=1}^{n} \epsilon_{Z_{t} / b(m)}(u, \infty] u^{\beta-1} d u \stackrel{P}{\rightarrow} \int_{0}^{x} \nu(u, \infty] u^{\beta-1} d u=\frac{x^{\beta-\alpha}}{\beta-\alpha} .
$$

(b) Suppose Condition $L$ holds so that $F \in R V(\alpha)$ at 0 . Then in $C_{0}[0, \infty)$ we have

$$
\int_{0}^{x} \nu_{n}\left[0, u^{-1}\right) u^{\beta-1} d u=\int_{0}^{x} \frac{m}{n} \sum_{t=1}^{n} \epsilon_{Z_{t} / a(m)}\left[0, u^{-1}\right) u^{\beta-1} d u \stackrel{P}{\rightarrow} \int_{0}^{x} \nu\left[0, u^{-1}\right) u^{\beta-1} d u=\frac{x^{\beta-\alpha}}{\beta-\alpha} .
$$

Proof. We first show (3.1) holds for any fixed $x$. Pick $\eta<x$. Since, for all $u>0$,

$$
\nu_{n}(u, \infty] \stackrel{P}{\rightarrow} \nu(u, \infty]
$$

we have

$$
\int_{\eta}^{x} \nu_{n}(u, \infty] u^{\beta-1} d u \stackrel{P}{\rightarrow} \int_{\eta}^{x} \nu(u, \infty] u^{\beta-1} d u .
$$

To prove (3.1) for the fixed value of $x$, we claim it is enough to show for all $\delta>0$ that

$$
\lim _{\eta \downarrow 0} \limsup _{n \rightarrow \infty} P\left[\int_{0}^{\eta} \nu_{n}(u, \infty] u^{\beta-1} d u>\delta\right]=0 .
$$

The reason (3.4) is sufficient can be argued as follows:

$$
\begin{aligned}
& P\left[\left|\int_{0}^{x} \nu_{n}(u, \infty] u^{\beta-1} d u-\int_{0}^{x} \nu(u, \infty] u^{\beta-1} d u\right|>\delta\right] \\
& \quad \leq P\left[\left|\int_{0}^{\eta} u^{\beta-1} \nu_{n}-\int_{0}^{\eta} u^{\beta-1} \nu\right|>\delta / 2\right]+P\left[\left|\int_{\eta}^{x} u^{\beta-1} \nu_{n}-\int_{\eta}^{x} u^{\beta-1} \nu\right|>\delta / 2\right] \\
& \quad=A_{n}+B_{n} .
\end{aligned}
$$

Now by (3.3) we have $B_{n} \rightarrow 0$. For $A_{n}$ we have

$$
A_{n}=P\left[\left|\int_{0}^{\eta} u^{\beta-1} \nu_{n}-\frac{\eta^{\beta-\alpha}}{\beta-\alpha}\right|>\delta / 2\right]
$$

and for $\eta$ much smaller than $\delta$ this is bounded by 


$$
\leq P\left[\int_{0}^{\eta} u^{\beta-1} \nu_{n}>\delta / 8\right]
$$

which goes to zero as $n \rightarrow \infty$ and then $\eta \downarrow 0$. Thus we conclude as desired

$$
\lim _{n \rightarrow \infty} P\left[\left|\int_{0}^{x} u^{\beta-1} \nu_{n}-\int_{0}^{x} u^{\beta-1} \nu\right|>\delta\right]=0
$$

and so we only need to verify (3.4).

For (3.4) apply Chebyshev's inequality:

$$
\begin{aligned}
P\left[\int_{0}^{\eta} \nu_{n}(u, \infty] u^{\beta-1} d u>\delta\right] & \leq \delta^{-1} E \int_{0}^{\eta} u^{\beta-1} \nu_{n} \\
& =\delta^{-1} \int_{0}^{\eta} E \nu_{n}(u, \infty] u^{\beta-1} d u=\delta^{-1} \int_{0}^{\eta} m P\left[\frac{Z_{1}}{b(m)}>u\right] u^{\beta-1} d u
\end{aligned}
$$

and applying Karamata's theorem, we find this converges as $n \rightarrow \infty$ to

$$
\rightarrow \delta^{-1} \int_{0}^{\eta} u^{-\alpha} u^{\beta-1} d u=\delta^{-1} \frac{\eta^{\beta-\alpha}}{\beta-\alpha}
$$

which converges to zero as $\eta \downarrow 0$.

This confirms (3.1) for fixed $x$. To verify the claim that convergence in probability takes place in $C_{0}[0, \infty)$ let $D$ be a countable dense subset of $[0, \infty)$. Write $(3.1)$ as $L_{n}(x) \stackrel{P}{\rightarrow} L(x)$ and we know this is true for a fixed $x$. It is no trouble obtaining

$$
\left\{L_{n}(x), x \in D\right\} \stackrel{P}{\rightarrow}\{L(x), x \in D\}
$$

in $\mathbb{R}^{\infty}$. Given any subsequence $\left\{n^{\prime \prime}\right\}$ there exists a further subsequence $\left\{n^{\prime}\right\} \subset\left\{n^{\prime \prime}\right\}$ such that

$$
\left\{L_{n^{\prime}}(x), x \in D\right\} \rightarrow\{L(x), x \in D\}
$$

almost surely in $\mathbb{R}^{\infty}$. Since the converging processes are monotone and the limit process is continuous we have local uniform convergence. Thus, by the subsequence characterization of convergence in probability in $C_{0}[0, \infty)$ the result follows.

To prove (b), we can use (a) since

$$
\int_{0}^{x} \frac{m}{n} \sum_{i=1}^{n} \epsilon_{Z_{i} / a(m)}\left[0, u^{-1}\right) u^{\beta-1} d u=\int_{0}^{x} \frac{m}{n} \sum_{i=1}^{n} \epsilon_{\frac{1 / Z_{i}}{1 / a(m)}}(u, \infty] u^{\beta-1} d u
$$

If $Z_{1}$ has a distribution which is regularly varying at 0 , then $1 / Z_{1}$ has a distribution which is regularly varying at $\infty$.

A more difficult result in this vein replaces $\left\{Z_{i}\right\}$ by the estimated non-zero residuals in the autoregression where the coefficients are estimated by the lp estimators.

Proposition 3.2. Suppose $\left\{X_{t} ; t=0, \pm 1, \pm 2, \ldots\right\}$ is the $A R(p)$ and suppose conditions $M$ and $S$ hold. Let $\hat{\phi}(n)$ be the lp estimators of the coefficients based on $\left\{X_{1}, \ldots, X_{n}\right\}$. Define the estimated residuals $\hat{Z}_{t}(n), t=p+1, \ldots, n$ and the residual empirical distribution $\hat{F}_{n}^{r e s i d}$ as in Section 1 (see also 2.11-2.15). Suppose $n \rightarrow \infty, m \rightarrow \infty, m / n \rightarrow 0$ as $n \rightarrow \infty$. 
(a) Suppose Condition $L$ holds and $\beta>\alpha$ and

$$
m\left(\frac{a(n)}{a(m)}\right)^{\beta} \rightarrow 0
$$

Then setting

$$
\hat{\nu}_{n}=\frac{m}{n-2 p} \sum_{t=p+1}^{n} 1_{\left[\hat{Z}_{t}(n)>0\right]} \epsilon_{\hat{Z}_{t}(n) / a(m)}=m \hat{F}_{n}^{r e s i d}(a(m) \cdot)
$$

we have

$$
\hat{\nu}_{n} \Rightarrow \nu
$$

in $M_{+}[0, \infty)$ where $\nu[0, u]=u^{\alpha}$ and furthermore in $C_{0}[0, \infty)$

$$
\int_{0}^{x} \hat{\nu}_{n}\left[0, u^{-1}\right] u^{\beta-1} d u \stackrel{P}{\rightarrow} \frac{x^{\beta-\alpha}}{\beta-\alpha} .
$$

(b) Suppose Condition $R$ holds and $\beta>\alpha$. Then $\hat{\nu}_{n}=m \hat{F}_{n}^{\text {resid }}(b(m) \cdot)$ satisfies

$$
\hat{\nu}_{n} \Rightarrow \nu
$$

in $M_{+}(0, \infty]$, where $\nu(u, \infty]=u^{-\alpha}, u>0$ and in $C_{0}[0, \infty)$,

$$
\int_{0}^{x} \hat{\nu}_{n}(u, \infty] u^{\beta-1} d u \stackrel{P}{\rightarrow} \int_{0}^{x} \nu(u, \infty] u^{\beta-1} d u=\frac{x^{\beta-\alpha}}{\beta-\alpha} .
$$

Proof. (a) The proof of (3.6) is given in Proposition 6.1 of Feigin, Resnick and Starica (1995). For (3.7), as in the proof of Proposition 3.1, it suffices to prove for any $\delta>0$

$$
\limsup _{\eta \downarrow 0} \operatorname{limsu}_{n \rightarrow \infty} P\left[\int_{0}^{\eta} \hat{\nu}_{n}\left[0, u^{-1}\right] u^{\beta-1} d u>\delta\right]=0 .
$$

For a large number $M$, define the event

$$
L E S S=\left[\frac{\|\hat{\phi}(n)-\phi(0)\|}{a(n)} \leq M\right] .
$$

Write the probability in (3.10) as

$$
\begin{gathered}
P\left[\int_{0}^{\eta} \hat{\nu}_{n}\left[0, u^{-1}\right] u^{\beta-1} d u>\delta\right] \\
\leq P\left[\int_{0}^{\eta} \hat{\nu}_{n}\left[0, u^{-1}\right] u^{\beta-1} d u 1_{L E S S}>\delta / 2\right] \\
\quad+P\left[\int_{0}^{\eta} \hat{\nu}_{n}\left[0, u^{-1}\right] u^{\beta-1} d u 1_{L E S S^{c}}>\delta / 2\right] \\
=A+B .
\end{gathered}
$$


Since we know (Feigin and Resnick, 1994) that there is a proper random vector $\mathbf{L}$ such that

$$
\frac{\|\hat{\phi}(n)-\phi(0)\|}{a(n)} \Rightarrow\|\mathbf{L}\|
$$

we have that

$$
B \leq P\left[\frac{\mid \hat{\phi}(n)-\phi(0) \|}{a(n)}>M\right] \rightarrow P[\|\mathbf{L}\|>M]
$$

as $n \rightarrow \infty$ and this last term can be made as small as one likes by appropriate choice of $M$. For $A$ we have the Chebyshev bound

$$
\begin{aligned}
A & \leq(\delta / 2)^{-1} E \int_{0}^{\eta} \frac{m}{n-2 p} \sum_{t=p+1}^{n} 1_{\left[\hat{Z}_{t}(n) / a(m) \leq u^{-1}, L E S S\right]} u^{\beta-1} d u \\
& =(\delta / 2)^{-1} \frac{m}{n-2 p} \sum_{t=p+1}^{n} \int_{0}^{\eta} P\left[\frac{\hat{Z}_{t}(n)}{a(m)} \leq u^{-1}, L E S S\right] u^{\beta-1} d u
\end{aligned}
$$

and for some small $\gamma>0$ we have the bound

$$
\begin{aligned}
& =(\delta / 2)^{-1} \frac{m}{n-2 p} \sum_{t=p+1}^{n} \int_{0}^{\eta}\left(P\left[\frac{\hat{Z}_{t}(n)}{a(m)} \leq u^{-1}, \frac{Z_{t}}{a(m)} \leq u^{-1}+\gamma, \text { LESS }\right]\right. \\
& \left.+P\left[\frac{\hat{Z}_{t}(n)}{a(m)} \leq u^{-1}, \frac{Z_{t}}{a(m)} \geq u^{-1}+\gamma, \text { LESS }\right]\right) u^{\beta-1} d u \\
& =A 1+A 2 .
\end{aligned}
$$

Now for small $\gamma$ and $\eta$

$$
\begin{aligned}
A 1 & \leq(\delta / 2)^{-1} \int_{0}^{\eta} m P\left[\frac{Z_{t}}{a(m)} \leq((1-\gamma) u)^{-1}\right] u^{\beta-1} d u \\
& =(\delta / 2)^{-1} \int_{0}^{(1-\gamma) \eta} m P\left[\frac{Z_{t}}{a(m)} \leq s^{-1}\right] \frac{s^{\beta-1} d s}{(1-\gamma)^{\beta}} \\
& \rightarrow(\text { const }) \eta^{\beta-\alpha},
\end{aligned}
$$

as $n \rightarrow \infty$ by Karamata's theorem. This last expression goes to zero as $\eta \downarrow 0$.

Finally, for $A 2$ we get the bound

$$
\begin{aligned}
A 2 & \leq(\delta / 2)^{-1} \frac{m}{n-2 p} \sum_{t=p+1}^{n} \int_{0}^{\eta} P\left[\frac{Z_{t}-\hat{Z}_{t}(n)}{a(m)}>\gamma, L E S S\right] u^{\beta-1} d u \\
& \leq(\delta / 2)^{-1} \frac{m}{n-2 p} \sum_{t=p+1}^{n} \int_{0}^{\eta} P\left[\|\phi(0)-\hat{\phi}(n)\| \frac{\left\|\mathbf{X}_{t-1}\right\|}{a(m)}>\gamma, L E S S\right] u^{\beta-1} d u \\
& \leq(\delta / 2)^{-1} m \frac{n-p}{n-2 p} \int_{0}^{\eta} P\left[a(n) M \frac{\left\|\mathbf{X}_{t-1}\right\|}{a(m)}>\gamma\right] u^{\beta-1} d u \\
& \leq(\text { const }) m\left(\frac{a(n)}{a(m)}\right)^{\beta} E\left\|\mathbf{X}_{1}\right\|^{\beta} \eta^{\beta} \rightarrow 0
\end{aligned}
$$


as $n \rightarrow \infty$, since $E\left\|\mathbf{X}_{1}\right\|^{\beta}<\infty$ follows from $E Z_{1}^{\beta}<\infty$ and from $(2.17-2.20)$

Proof of (b): The proof of (3.8) is essentially given in Proposition 4.1 of Resnick and Starica (1995). A slight adaptation of that result is used here. In effect, we do not need to restrict the observed residuals $\hat{Z}_{t}(n)$ to those for which $\mathbf{X}_{t-1} \in[0, \infty]^{p} \backslash\{\mathbf{0}\}$ when using the residuals based on the lp estimators; this follows from the fact that $\hat{Z}_{t}(n) \geq 0$.

To prove (3.9), we again need to show (3.10). For any real number $M$, we define the event

$$
L E S S=[\|\hat{\phi}(n)-\phi(0)\| \leq M]
$$

Then we have

$$
\begin{aligned}
& P\left[\int_{0}^{\eta} \hat{\nu}_{n}(u, \infty] u^{\beta-1} d u>\delta\right] \\
& \quad=P\left[\int_{0}^{\eta} \hat{\nu}_{n}(u, \infty] u^{\beta-1} d u 1_{[L E S S]}>\delta / 2\right]+P\left[\int_{0}^{\eta} \hat{\nu}_{n}(u, \infty] u^{\beta-1} d u 1_{[L E S S c]}>\delta / 2\right] \\
& \quad=A+B .
\end{aligned}
$$

Now $B$ is easily handled since

$$
B \leq P[\|\hat{\phi}(n)-\phi(0)\|>M] \rightarrow 0
$$

as $n \rightarrow \infty$. For $A$ we have the bound ( $\xi>0$ is small)

$$
\begin{aligned}
& A \leq\left(\frac{\delta}{2}\right)^{-1} E \int_{0}^{\eta} \frac{m}{n-2 p} \sum_{t=p+1}^{n} \epsilon_{\hat{Z}_{t}(n) / b(m)}(u, \infty] 1_{[L E S S]} u^{\beta-1} d u \\
& =\left(\frac{\delta}{2}\right)^{-1} \frac{m}{n-2 p} \sum_{t=p+1}^{n} \int_{0}^{\eta} P\left[\hat{Z}_{t}(n) / b(m)>u, L E S S\right] u^{\beta-1} d u \\
& =\left(\frac{\delta}{2}\right)^{-1} \frac{m}{n-2 p} \sum_{t=p+1}^{n} \int_{0}^{\eta} P\left[\hat{Z}_{t}(n) / b(m)>u, Z_{t} / b(m)>u(1-\xi), L E S S\right] u^{\beta-1} d u \\
& \quad+\left(\frac{\delta}{2}\right)^{-1} \frac{m}{n-2 p} \sum_{t=p+1}^{n} \int_{0}^{\eta} P\left[\hat{Z}_{t}(n) / b(m)>u, Z_{t} / b(m) \leq u(1-\xi), L E S S\right] u^{\beta-1} d u \\
& =A 1+A 2 .
\end{aligned}
$$

We deal with the pieces separately. For $A 1$ we have

$$
\begin{aligned}
A 1 & \leq\left(\frac{\delta}{2}\right)^{-1} \frac{m}{n-2 p} \sum_{t=p+1}^{n} \int_{0}^{\eta} P\left[Z_{t} / b(m)>u(1-\xi)\right] u^{\beta-1} d u \\
& =\left(\frac{\delta}{2}\right)^{-1} \frac{n-p}{n-2 p} \int_{0}^{\eta(1-\xi)} m P\left[Z_{1} / b(m)>u\right] u^{\beta-1} d u /(1-\xi)^{\beta}
\end{aligned}
$$

and applying Karamata's theorem this converges, as $n \rightarrow \infty$, to

$$
\begin{aligned}
& \rightarrow\left(\frac{\delta}{2}\right)^{-1} \int_{0}^{\eta(1-\xi)} u^{\beta-\alpha-1} d u /(1-\xi)^{\beta} \\
& \rightarrow 0
\end{aligned}
$$


as $\eta \downarrow 0$.

Finally we control $A 2$. Recall, from (2.15), on $L E S S$ we have the bound $\left|\hat{Z}_{t}(n)-Z_{t}\right| \leq M\left\|\mathbf{X}_{t-1}\right\|$. Therefore, using (2.18),

$$
\begin{aligned}
A 2 & \leq\left(\frac{\delta}{2}\right)^{-1} \frac{m}{n-2 p} \sum_{t=p+1}^{n} \int_{0}^{\eta} P\left[M\left\|\mathbf{X}_{t-1}\right\| / b(m)>u \xi\right] u^{\beta-1} d u \\
& \leq\left(\frac{\delta}{2}\right)^{-1} \frac{n-p}{n-2 p} \int_{0}^{\eta} m P\left[\left\|\mathbf{X}_{1}\right\| / b(m)>u \xi\right] u^{\beta-1} d u \\
& \leq\left(\frac{\delta}{2}\right)^{-1} \frac{n-p}{n-2 p} \int_{0}^{\eta} m P\left[\sum_{j=0}^{\infty}\left\|\mathbf{c}_{j}\right\| Z_{j} / b(m)>u \xi\right] u^{\beta-1} d u
\end{aligned}
$$

and because of a result of Cline (1983) (see an account in Resnick, 1987, page 227), $\sum_{j=0}^{\infty}\left\|\mathbf{c}_{j}\right\| Z_{j}$ has regularly varying tail probabilities and so we get by Karamata's theorem, as $n \rightarrow \infty$

$$
\begin{aligned}
& \rightarrow\left(\frac{\delta}{2}\right)^{-1} \int_{0}^{\eta} \sum_{j=0}^{\infty}\left\|\mathbf{c}_{j}\right\|^{\alpha}(u \xi)^{-\alpha} u^{\beta-1} d u \\
& \rightarrow 0
\end{aligned}
$$

as $\eta \downarrow 0$. This completes the proof.

For treating the right tailed case, we need the following series versions of the previous results.

Proposition 3.3. Suppose $\left\{\psi_{j}(n), j \geq 0, n \geq 0\right\}$ is any collection of non-negative real numbers such that for some $0<\delta<\alpha \wedge 1$

$$
\begin{aligned}
& \sum_{j=0}^{\infty} \psi_{j}(n)^{\delta}<\infty, \quad n \geq 0, \\
& \lim _{n \rightarrow \infty} \psi_{j}(n)=\psi_{j}(0), \quad j \geq 0, \\
& \lim _{q \rightarrow \infty} \limsup _{n \rightarrow \infty} \sum_{j=q}^{\infty} \psi_{j}(n)^{\delta}=0 .
\end{aligned}
$$

Suppose $F$ satisfies the regular variation Condition $R(1.8)$. Then for $x>1$, as $n \rightarrow \infty$

$$
\begin{aligned}
& \text { (1) } \sum_{j=0}^{\infty} m\left(1-F\left(b(m) \psi_{j}(n)^{-1} x\right)\right) \rightarrow \sum_{j=0}^{\infty} \psi_{j}(0)^{\alpha} x^{-\alpha} \\
& \text { (2) } \sum_{j=0}^{\infty} \int_{0}^{x} u^{\beta-1} m\left(1-F\left(b(m) \psi_{j}(n)^{-1} u\right)\right) d u \rightarrow \sum_{j=0}^{\infty} \psi_{j}(0)^{\alpha} \frac{x^{\beta-\alpha}}{\beta-\alpha}
\end{aligned}
$$

Suppose, in addition, that $\left\{X_{t} ; t=0, \pm 1, \pm 2, \ldots\right\}$ is an $A R(p)$ process satisfying conditions $M, S$ and $R$. Then

$$
\text { (3) } \sum_{j=0}^{\infty} m\left(1-\hat{F}_{n}^{r e s i d}\left(b(m) \psi_{j}(n)^{-1} x\right)\right) \stackrel{P}{\rightarrow} \sum_{j=0}^{\infty} \psi_{j}(0)^{\alpha} x^{-\alpha}
$$

for $x>0$, 


$$
\text { (4) } \sum_{j=0}^{\infty} \int_{0}^{x} u^{\beta-1} m\left(1-\hat{F}_{n}^{r e s i d}\left(b(m) \psi_{j}(n)^{-1} u\right)\right) d u \stackrel{P}{\rightarrow} \sum_{j=0}^{\infty} \psi_{j}(0)^{\alpha} \frac{x^{\beta-\alpha}}{\beta-\alpha},
$$

in $C_{0}[0, \infty)$.

Proof. (1) For every fixed $j$, the uniform convergence inherent in regular variation implies

$$
m\left(1-F\left(b(m) \psi_{j}(n)^{-1} x\right)\right) \rightarrow \psi_{j}(0)^{\alpha} x^{-\alpha}
$$

and hence for any integer $q$ we have

$$
m \sum_{j=0}^{q}\left(1-F\left(b(m) \psi_{j}(n)^{-1} x\right)\right) \rightarrow \sum_{j=0}^{q} \psi_{j}(0)^{\alpha} x^{-\alpha} .
$$

It suffices to show

$$
\lim _{q \rightarrow \infty} \limsup _{n \rightarrow \infty} \sum_{j=q+1}^{\infty} m\left(1-F\left(b(m) \psi_{j}(n)^{-1} x\right)\right)=0 .
$$

Write

$$
\begin{aligned}
& \sum_{j=q+1}^{\infty} m\left(1-F\left(b(m) \psi_{j}(n)^{-1} x\right)\right)=\sum_{j: j>q, \psi_{j}(n) \leq 1}+\sum_{j: j>q, \psi_{j}(n)>1} \\
& =A+B \text {. }
\end{aligned}
$$

Now

$$
A=\sum_{j: j>q, \psi_{j}(n) \leq 1}\left(\frac{1-F\left(b(m) \psi_{j}(n)^{-1} x\right)}{1-F(b(m))}\right)
$$

and applying Potter's inequality (Bingham et.al., 1987, page 21) we get for any $\epsilon$ small, $0<\delta<\alpha \wedge 1$, and large enough $n$ that the above is bounded by

$$
\leq \sum_{j: j>q, \psi_{j}(n) \leq 1} \psi_{j}(n)^{\delta} x^{-(\alpha-\epsilon)}
$$

and hence, from (3.13),

$$
\lim _{q \rightarrow \infty} \limsup _{n \rightarrow \infty} A=0
$$

For $B$ observe that since

$$
\lim _{q \rightarrow \infty} \limsup _{n \rightarrow \infty} \sum_{j=q}^{\infty} \psi_{j}(n)^{\delta}=0,
$$

given $\epsilon<1 / 2$, there exists $q_{0}=q_{0}(\epsilon)$ such that

$$
\limsup _{n \rightarrow \infty} \sum_{j=q_{0}}^{\infty} \psi_{j}(n)^{\delta}<\epsilon
$$


and hence there exists $n_{0}=n_{0}\left(q_{0}(\epsilon), \epsilon\right)$ such that for $n \geq n_{0}$

$$
\sum_{j=q_{0}}^{\infty} \psi_{j}(n)^{\delta}<2 \epsilon
$$

Therefore,

$$
N_{n}:=\#\left\{j: \psi_{j}(n)>1\right\}<q_{0}
$$

Thus for $B$, we have

$$
B \leq \sum_{q+1}^{q_{0}} m\left(1-F\left(b(m) \psi_{j}(n)^{-1} x\right)\right)=0
$$

if $q \geq q_{0}$.

(2) For any fixed $j$, we have by Karamata's theorem that

$$
\int_{0}^{x} u^{\beta-1} m\left(1-F\left(b(m) \psi_{j}(n)^{-1} u\right)\right) d u \rightarrow \psi_{j}(0)^{\alpha} \frac{x^{\beta-\alpha}}{\beta-\alpha}
$$

and so for any $q$

$$
\sum_{j=0}^{q} \int_{0}^{x} u^{\beta-1} m\left(1-F\left(b(m) \psi_{j}(n)^{-1} u\right)\right) d u \rightarrow \sum_{j=0}^{q} \psi_{j}(0)^{\alpha} \frac{x^{\beta-\alpha}}{\beta-\alpha}
$$

Thus it suffices to show

$$
\limsup _{q \rightarrow \infty} \limsup _{n \rightarrow \infty} \sum_{j=q+1}^{x} u_{0}^{\beta-1} m\left(1-F\left(b(m) \psi_{j}(n)^{-1} u\right)\right) d u=0 .
$$

Observe that

$$
\begin{array}{rl}
\int_{0}^{x} u^{\beta-1} & m\left(1-F\left(b(m) \psi_{j}(n)^{-1} u\right)\right) d u \\
= & \frac{m \psi_{j}(n)^{\beta}}{b(m)^{\beta}} \int_{0}^{b(m) \psi_{j}(n)^{-1} x} s^{\beta-1}(1-F(s)) d s \\
= & \psi_{j}(n)^{\beta}\left(\frac{\int_{0}^{b(m) \psi_{j}(n)^{-1} x} s^{\beta-1}(1-F(s)) d s}{\int_{0}^{b(m)} s^{\beta-1}(1-F(s)) d s}\right)\left(\frac{m \int_{0}^{b(m)} s^{\beta-1}(1-F(s)) d s}{b(m)^{\beta}}\right) .
\end{array}
$$

Suppose $\psi_{j}(n) \leq 1$. Then by Potter's inequalities, for any $\eta$ and for $n$ sufficiently large we have the upper bound on the last expression of

$$
\psi_{j}(n)^{\beta}\left(\psi_{j}(n)^{-1} x\right)^{\beta-\alpha+\eta} \frac{m \int_{0}^{b(m)} s^{\beta-1}(1-F(s)) d s}{b(m)^{\beta}} .
$$

However, by Karamata's theorem,

$$
\frac{m \int_{0}^{b(m)} s^{\beta-1}(1-F(s)) d s}{b(m)^{\beta}} \rightarrow \frac{1}{\beta-\alpha}
$$


and so for some constant $K>0$ we have

$$
\begin{array}{rl}
\sum_{j: j>q, \psi_{j}(n) \leq 1} \int_{0}^{x} u^{\beta-1} & m\left(1-F\left(b(m) \psi_{j}(n)^{-1} u\right)\right) d u \\
\leq K & \sum_{j: j>q, \psi_{j}(n) \leq 1} \psi_{j}(n)^{\beta-(\beta-\alpha+\eta)} x^{\beta-\alpha+\eta} \\
& =K \sum_{j: j>q, \psi_{j}(n) \leq 1} \psi_{j}(n)^{\alpha-\eta} x^{\beta-\alpha+\eta} .
\end{array}
$$

Choose $\eta$ to satisfy $\alpha-\eta=\delta$, that is, $\alpha-\delta=\eta$ and the bound is

$$
K \sum_{j: j>q, \psi_{j}(n) \leq 1} \psi_{j}(n)^{\delta} x^{\beta-\delta}
$$

Hence

$$
\lim _{q \rightarrow \infty} \limsup _{n \rightarrow \infty} \sum_{j: j>q, \psi_{j}(n) \leq 1} \int_{0}^{x} u^{\beta-1} m\left(1-F\left(b(m) \psi_{j}(n)^{-1} u\right)\right) d u=0,
$$

and the proof is finished as in part (1).

(3) We know from (3.8) that for any $j$

$$
m \hat{F}_{n}^{r e s i d}\left(b(m) \psi_{j}(n)^{-1} x, \infty\right] \stackrel{P}{\rightarrow} \psi_{j}(0)^{\alpha} x^{-\alpha},
$$

so for any $q$,

$$
\sum_{j=0}^{q} m \hat{F}_{n}^{r e s i d}\left(b(m) \psi_{j}(n)^{-1} x, \infty\right] \stackrel{P}{\rightarrow} \sum_{j=0}^{q} \psi_{j}(0)^{\alpha} x^{-\alpha},
$$

so it suffices to show that for any $\eta>0$

$$
\lim _{q \rightarrow \infty} \limsup _{n \rightarrow \infty} P\left[\sum_{j=q+1}^{\infty} m \hat{F}_{n}^{r e s i d}\left(b(m) \psi_{j}(n)^{-1} x, \infty\right]>\eta\right]=0 .
$$

Define for $M>0$

$$
[L E S S]=[\|\hat{\phi}(n)-\phi(0)\| \leq M]
$$

so that by consistency of the lp estimators we have $P\left([L E S S]^{c}\right) \rightarrow 0$. Then

$$
\begin{aligned}
P\left[\sum_{j=q+1}^{\infty} m \hat{F}_{n}^{r e s i d}\left(b(m) \psi_{j}(n)^{-1} x, \infty\right]>\eta\right] \\
\leq P\left([L E S S]^{c}\right)+P\left[\sum_{j=q+1}^{\infty} m \hat{F}_{n}^{r e s i d}\left(b(m) \psi_{j}(n)^{-1} x, \infty\right] 1_{[L E S S]}>\eta / 2\right] \\
=A+B .
\end{aligned}
$$

Now $A \rightarrow 0$ as $n \rightarrow \infty$. For $B$ we have

$$
P\left[\sum_{j=q+1}^{\infty} m \frac{1}{n-2 p} \sum_{i=p+1}^{n} 1_{\left[\hat{Z}_{i}(n)>b(m) \psi_{j}(n)^{-1} x, L E S S\right]}>\eta / 2\right]
$$


which has a Chebyshev bound of

$$
\sum_{j=q+1}^{\infty} m \frac{1}{n-2 p} \sum_{i=p+1}^{n} P\left[\hat{Z}_{i}(n)>b(m) \psi_{j}(n)^{-1} x, L E S S\right] /(\eta / 2)
$$

which for any small $\chi$ is bounded by

$$
\begin{aligned}
& \frac{2}{\eta} \sum_{j=q+1}^{\infty} \frac{m}{n-2 p} \sum_{i=p+1}^{n} P\left[\hat{Z}_{i}(n)>b(m) \psi_{j}(n)^{-1} x, Z_{i}>b(m) \psi_{j}(n)^{-1}(1-\chi) x\right] \\
& \quad+\frac{2}{\eta} \sum_{j=q+1}^{\infty} \frac{m}{n-2 p} \sum_{i=p+1}^{n} P\left[\hat{Z}_{i}(n)>b(m) \psi_{j}(n)^{-1} x, Z_{i} \leq b(m) \psi_{j}(n)^{-1}(1-\chi) x, L E S S\right] \\
& =B 1+B 2 .
\end{aligned}
$$

Now from part (1)

$$
\begin{aligned}
B 1 & \leq \frac{2}{\eta} \sum_{j=q+1}^{\infty} m P\left[Z_{1}>b(m) \psi_{j}(n)^{-1}(1-\chi) x\right] \\
& \rightarrow \frac{2}{\eta} \sum_{j=q+1}^{\infty}(x(1-\chi))^{-\alpha} \psi_{j}(0)^{\alpha}
\end{aligned}
$$

as $n \rightarrow \infty$ and as $q \rightarrow \infty$, this converges to zero. For $B 2$ we get by recalling (2.14), that

$$
\left|\hat{Z}_{t}(n)-Z_{t}\right|=\left|(\hat{\phi}(n)-\phi(0))^{\prime} \mathbf{X}_{t-1}\right| .
$$

Thus

$$
\begin{aligned}
B 2 & \leq \frac{2}{\eta} \sum_{j=q+1}^{\infty} \frac{m}{n-2 p} \sum_{t=p+1}^{n} P\left[\left|\hat{Z}_{t}(n)-Z_{t}\right|>b(m) \psi_{j}(n)^{-1} \chi x, L E S S\right] \\
& \leq \frac{2}{\eta} \sum_{j=q+1}^{\infty} \frac{m}{n-2 p} \sum_{t=p+1}^{n} P\left[\|\hat{\phi}(n)-\boldsymbol{\phi}(0)\|\left\|\mathbf{X}_{t-1}\right\|>b(m) \psi_{j}(n)^{-1} \chi x, L E S S\right] \\
& \leq \frac{2}{\eta} \frac{n-p}{n-2 p} \sum_{j=q+1}^{\infty} m P\left[M\left\|\mathbf{X}_{t-1}\right\|>b(m) \psi_{j}(n)^{-1} \chi x\right] \\
& \leq \frac{2}{\eta} \frac{n-p}{n-2 p} \sum_{j=q+1}^{\infty} m P\left[\left\|\mathbf{X}_{1}\right\|>b(m) \psi_{j}(n)^{-1} \chi x M^{-1}\right] .
\end{aligned}
$$

Write $\mathbf{X}_{t}=\sum_{j=0}^{\infty} \mathbf{c}_{j} Z_{t-j}$ so that (see 2.19) $\left\|\mathbf{X}_{t}\right\| \leq \sum_{j=0}^{\infty}\left\|\mathbf{c}_{j}\right\| Z_{t-j}$ and we obtain

$$
B 2 \leq \frac{2}{\eta} \frac{n-p}{n-2 p} \sum_{j=q+1}^{\infty} m P\left[\sum_{k=0}^{\infty}\left\|\mathbf{c}_{k}\right\| Z_{k}>b(m) \psi_{j}(n)^{-1} \chi x M^{-1}\right]
$$

and since $\sum_{j=0}^{\infty}\left\|\mathbf{c}_{j}\right\| Z_{j}$ is a random variable whose distribution has a tail which is regularly varying with index $-\alpha$, we get from part (1) that

$$
\lim _{q \rightarrow \infty} \limsup _{n \rightarrow \infty} B 2=0 .
$$


(4) For any fixed $j$ we have from (3.9) (recall that (3.9) is convergence in $C_{0}[0, \infty)$ ) that

$$
\int_{0}^{x} u^{\beta-1} m \hat{F}_{n}^{r e s i d}\left(b(m) \psi_{j}(n)^{-1} u, \infty\right] d u \stackrel{P}{\rightarrow} \psi_{j}(0)^{\alpha} \frac{x^{\beta-\alpha}}{\beta-\alpha}
$$

and hence for any $q$

$$
\sum_{j=0}^{q} \int_{0}^{x} u^{\beta-1} m \hat{F}_{n}^{r e s i d}\left(b(m) \psi_{j}(n)^{-1} u, \infty\right] d u \stackrel{P}{\rightarrow} \sum_{j=0}^{q} \psi_{j}(0)^{\alpha} \frac{x^{\beta-\alpha}}{\beta-\alpha} .
$$

So it suffices to show for any $\eta>0$

$$
\limsup _{q \rightarrow \infty} \limsup _{n \rightarrow \infty} P\left[\sum_{j=q+1}^{\infty} \int_{0}^{x} u^{\beta-1} m \hat{F}_{n}^{r e s i d}\left(b(m) \psi_{j}(n)^{-1} u, \infty\right] d u>\eta\right]=0 .
$$

However the probability on the previous line is bounded by

$$
\begin{aligned}
P\left[\sum_{j=q+1}^{\infty} \int_{0}^{x} u^{\beta-1} m \hat{F}_{n}^{r e s i d}\left(b(m) \psi_{j}(n)^{-1} u, \infty\right] d u 1_{[L E S S]}>\eta / 2\right] \\
+P\left[\sum_{j=q+1}^{\infty} \int_{0}^{x} u^{\beta-1} m \hat{F}_{n}^{r e s i d}\left(b(m) \psi_{j}(n)^{-1} u, \infty\right] d u 1_{[L E S S]^{c}}>\eta / 2\right] \\
=A+B .
\end{aligned}
$$

We quickly dispose of $B$ since $B \leq P\left([L E S S]^{c}\right) \rightarrow 0$ as $n \rightarrow \infty$. For $A$ we have

$$
\begin{aligned}
A \leq & \frac{2}{\eta} \sum_{j=q+1}^{\infty} \frac{m}{n-2 p} \int_{0}^{x} u^{\beta-1} E \sum_{i=p+1}^{n} 1_{\left[\hat{Z}_{i}(n) / b(m)>\psi_{j}(n)^{-1} u, L E S S\right]} d u \\
\leq & \frac{2}{\eta} \sum_{j=q+1}^{\infty} \frac{m}{n-2 p} \sum_{i=p+1}^{n} \int_{0}^{x} u^{\beta-1} P\left[\hat{Z}_{i}(n) / b(m)>\psi_{j}(n)^{-1} u, Z_{i}>b(m) \psi_{j}(n)^{-1} u(1-\chi)\right] d u \\
& \quad+\frac{2}{\eta} \sum_{j=q+1}^{\infty} \frac{m}{n-2 p} \sum_{i=p+1}^{n} \int_{0}^{x} u^{\beta-1} P\left[\hat{Z}_{i}(n) / b(m)>\psi_{j}(n)^{-1} u, Z_{i} \leq b(m) \psi_{j}(n)^{-1} u(1-\chi), L E S S\right] d u \\
= & A 1+A 2 .
\end{aligned}
$$

Now

$$
\begin{aligned}
A 1 & \leq \frac{2}{\eta} \sum_{j=q+1}^{\infty} \frac{m}{n-2 p} \sum_{i=p+1}^{n} \int_{0}^{x} u^{\beta-1} P\left[Z_{i}>b(m) \psi_{j}(n)^{-1} u(1-\chi)\right] d u \\
& \leq \frac{2}{\eta} \frac{n-p}{n-2 p} \sum_{j=q+1}^{\infty} \int_{0}^{x} u^{\beta-1} m P\left[Z_{i}>b(m) \psi_{j}(n)^{-1} u(1-\chi)\right] d u \\
& \rightarrow \frac{2}{\eta} \frac{n-p}{n-2 p} \sum_{j=q+1}^{\infty}\left(\psi_{j}(0)^{-1}(1-\chi)\right)^{-\alpha} \frac{x^{\beta-\alpha}}{\beta-\alpha}
\end{aligned}
$$

as $n \rightarrow \infty$ from (2). Thus $\lim _{q \rightarrow \infty} \lim \sup _{n \rightarrow \infty} A 1=0$. 
For $A 2$ we again note that $\hat{Z}_{t}(n)-Z_{t}=-(\hat{\phi}(n)-\phi(0))^{\prime} \mathbf{X}_{t-1}$ and continue

$$
\begin{aligned}
A 2 & \leq \frac{2}{\eta} \sum_{j=q+1}^{\infty} \int_{0}^{x} u^{\beta-1} \frac{m}{n-2 p} \sum_{t=p+1}^{n} P\left[\left|\hat{Z}_{t}(n)-Z_{t}\right|>b(m) \psi_{j}(n)^{-1} \chi u, L E S S\right] d u \\
& \leq \frac{2}{\eta} \sum_{j=q+1}^{\infty} \int_{0}^{x} u^{\beta-1} \frac{m}{n-2 p} \sum_{t=p+1}^{n} P\left[\|\hat{\boldsymbol{\phi}}(n)-\hat{\phi}(0)\|\left\|\mathbf{X}_{t-1}\right\|>b(m) \psi_{j}(n)^{-1} \chi u, L E S S\right] d u \\
& \leq \frac{2}{\eta} \sum_{j=q+1}^{\infty} \int_{0}^{x} u^{\beta-1} m \frac{n-p}{n-2 p} P\left[M\left\|\mathbf{X}_{1}\right\|>b(m) \psi_{j}(n)^{-1} \chi u p^{-1}\right] d u \\
& \leq \frac{2}{\eta} \sum_{j=q+1}^{\infty} \int_{0}^{x} u^{\beta-1} m P\left[\sum_{j=0}^{\infty}\left\|\mathbf{c}_{j}\right\| Z_{-j}>b(m) \psi_{j}(n)^{-1} \chi u M^{-1} p^{-1}\right] d u
\end{aligned}
$$

and because $\sum_{j=0}^{\infty}\left\|\mathbf{c}_{j}\right\| Z_{-j}$ has a distribution tail which is regularly varying (Cline, 1983) we get from (2) that

$$
\lim _{q \rightarrow \infty} \limsup _{n \rightarrow \infty} A 2=0
$$

There is some question how restrictive the hypotheses (3.11)-(3.13) of Proposition 3.3 are and it is reassuring to know that in the cases of interest, these are no problem to check. When we have a sequence of $\operatorname{AR}(p)$ processes expressed in $\mathrm{MA}(\infty)$ form, if the autoregressive coefficients converge, then the conditions of Proposition 3.3 hold. This is discussed next.

Proposition 3.4. Let $\phi(n)=\left(\phi_{1}(n), \phi_{2}(n), \ldots, \phi_{p}(n)\right)^{\prime}$ be vectors in $\mathbb{R}^{p}$ satisfying for $n \geq 0$

$$
\Phi_{n}(z):=1-\sum_{i=1}^{p} \phi_{i}(n) z^{i}
$$

has no roots in $|z| \leq 1$ and $\phi(n) \rightarrow \phi(0)$. Define

$$
C_{n}(z)=\frac{1}{\Phi_{n}(z)}=\sum_{j=0}^{\infty} c_{j}(n) z^{j},|z| \leq 1 ; \mathbf{c}_{j}(n)=\left(c_{j}(n), \ldots, c_{j-p+1}(n)\right)^{\prime} \text { with } c_{j}(n)=0, j<0 .
$$

Then we have for $0<\delta$

$$
\begin{aligned}
& \sum_{j=0}^{\infty}\left\|\mathbf{c}_{j}(n)\right\|^{\delta}<\infty, \quad n \geq 0, \\
& \lim _{n \rightarrow \infty} \mathbf{c}_{j}(n)=\mathbf{c}_{j}(0), \quad j \geq 0, \\
& \lim _{q \rightarrow \infty} \limsup _{n \rightarrow \infty} \sum_{j=q}^{\infty}\left\|\mathbf{c}_{j}(n)\right\|^{\delta}=0 .
\end{aligned}
$$

Proof. There exists $\epsilon>0$ such that

$$
\Phi_{0}(z) \neq 0, \quad|z| \leq 1+2 \epsilon .
$$

There exists $n_{0}=n_{0}(\epsilon)$ such that if $n \geq n_{0}$ or $n=0$ then

$$
\Phi_{n}(z) \neq 0, \quad|z| \leq 1+\epsilon
$$


and $\Phi_{n}(z) \rightarrow \Phi_{0}(z)$ uniformly in $|z| \leq 1+\epsilon$. Thus $C_{n}(z) \rightarrow C_{0}(z)$ uniformly in $|z| \leq 1+\epsilon$. Since for $j=0,1, \ldots$

$$
c_{j}(n)(1+\epsilon)^{j}=\frac{1}{2 \pi} \int_{-\pi}^{\pi} C_{n}\left((1+\epsilon) e^{i \theta}\right) e^{-i j \theta} d \theta,
$$

(Rudin, 1966, page 213) we have $c_{j}(n) \rightarrow c_{j}(0)$ as $n \rightarrow \infty$. For $n$ sufficiently large

$$
\begin{aligned}
\left|c_{j}(n)\right| & \leq(1+\epsilon)^{-j} \frac{1}{2 \pi} \int_{-\pi}^{\pi}\left|C_{n}\left((1+\epsilon) e^{i \theta}\right)\right| d \theta \\
& \leq(1+\epsilon)^{-j} \sup _{-\pi<\theta \leq \pi}\left|C_{n}\left((1+\epsilon) e^{i \theta}\right)\right| \\
& \leq 2(1+\epsilon)^{-j} \sup _{-\pi<\theta \leq \pi}\left|C_{0}\left((1+\epsilon) e^{i \theta}\right)\right| .
\end{aligned}
$$

The desired results now easily follow.

\section{The triangular array result underpinning the bootstrap.}

As we discussed in Section 2, in order to prove the asymptotic validity of the bootstrap, a triangular array generalization of a limit theorem stated for a single sequence is needed. This pattern is followed for instance in the papers of Datta and McCormick (1995) and Deheuvels, Mason and Shorack (1993).

We first give the triangular array theorem that we will need in the left tail case and follow this by a discussion of the right tail case.

Theorem 4.1. Suppose $\left\{Z_{t}\right\}$ (iid $F$ ) and $\left\{X_{t}\right\}$ satisfy the autoregression (1.1) and $F$ and $\phi$ satisfy conditions $M, S$ and $L$ with $a(n)$ the quantile function $F^{\leftarrow}(1 / n)$ as before.

Suppose $\Lambda \subset\{0,1, \ldots\}$ is unbounded. We will understand all limits as going to infinity through points in $\Lambda$ and all suprema as being taken over indices in $\Lambda$. As usual, $m=m(n)$ is a sequence going to $\infty$ such that $m / n \rightarrow 0$.

For each $n \in \Lambda$, suppose $\left\{Z_{i}(n), i \geq 1\right\}$ is iid with common distribution $F_{n}$. Let $F_{n}$ satisfy the following conditions:

(1) $F_{n}$ approximates $F$ weakly:

$$
F_{n} \Rightarrow F, \quad n \rightarrow \infty, n \in \Lambda
$$

(2) $F_{n}$ approximates $\nu$ vaguely in the sense that

$$
m F_{n}(a(m) \cdot) \stackrel{v}{\rightarrow} \nu
$$

in $M_{+}[0, \infty)$ where $\nu[0, x]=x^{\alpha}$.

(3) For some $\beta>\alpha$ :

$$
\bigvee_{n \in \Lambda} \int_{0}^{\infty} t^{\beta} F_{n}(d t)<\infty
$$

Suppose we have a sequence of vectors $\phi(n) \in \mathbb{R}^{p}, n \in \Lambda$ such that $\phi(n) \rightarrow \phi$ and let $\Phi_{n}(z)$ and $C_{n}(z)$ be defined as in Proposition 3.4.

Define $\lambda_{m}\left(F_{n}, \phi(n)\right)$ as in equations $(2.2-2.5)$. Then

$$
\lambda_{m}\left(F_{n}, \phi(n)\right)=\sum_{i=1}^{m} \epsilon_{\left(Z_{t}(n) / a(m), \mathbf{X}_{t-1}(n)\right)} \Rightarrow \sum_{k} \epsilon_{\left(j_{k}, \mathbf{Y}_{k}\right)}=\lambda_{\infty},
$$


in $M_{p}\left([0, \infty) \times([-\infty, \infty] \backslash\{0\})^{p}\right)$ where $\sum_{k} \epsilon_{j_{k}}$ is a Poisson process on $[0, \infty)$ with mean measure $\nu$ and $\left\{\mathbf{Y}_{k}\right\}$ is iid, independent of $\left\{j_{k}\right\}$ and

$$
\mathbf{Y}_{1} \stackrel{d}{=} \mathbf{X}_{p}=\left(X_{p}, \ldots, X_{1}\right)
$$

Furthermore if

$$
\lim _{\eta \downarrow 0} \limsup _{n \rightarrow \infty} m E\left(\frac{Z_{1}(n)}{a(m)}\right)^{-\beta} 1_{\left[\left(Z_{1}(n) / a(m)\right)^{-1} \leq \eta\right]}=0
$$

then

$$
T \lambda_{m}\left(F_{n}, \phi(n)\right)=\sum_{t=1}^{m} \epsilon_{a(m) Z_{t}(n)^{-1} \mathbf{X}_{t-1}} \Rightarrow \sum_{k} \epsilon_{j_{k}^{-1} \mathbf{Y}_{k}}
$$

in $M_{p}\left(([-\infty, \infty] \backslash\{0\})^{p}\right)$.

Proof. From (4.2) and Proposition 2.1 (see Proposition 3.21, Resnick, 1987) we get

$$
\sum_{t=1}^{m} \epsilon_{Z_{t}(n) / a(m)} \Rightarrow \sum_{k} \epsilon_{j_{k}}
$$

in $M_{p}[0, \infty)$ where the limit is $\operatorname{PRM}(\nu)$. From (2.3) and (2.5) we recall

$$
X_{t}(n)=\sum_{j=1}^{p} \phi_{j}(n) X_{t-j}(n)+Z_{t}(n) \text { and } \mathbf{X}_{t}(n)=\sum_{j=0}^{\infty} \mathbf{c}_{j}(n) Z_{t-j}(n)
$$

and define, similarly to (2.17),

$$
\mathbf{X}_{t, q}(n)=\sum_{j=0}^{q} \mathbf{c}_{j}(n) Z_{t-j}(n)
$$

We note also that, just as in $(2.19,2.20)$,

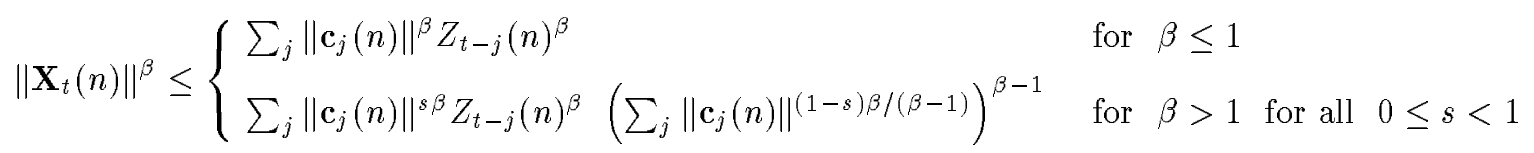

and as a result of Proposition 3.4 and condition (4.3)

$$
E\left\|\mathbf{X}_{t}(n)\right\|^{\beta}<\infty \text { and } \lim _{q \rightarrow \infty} \limsup _{n \rightarrow \infty} E\left\|\mathbf{X}_{t}(n)-\mathbf{X}_{t, q}(n)\right\|^{\beta} \rightarrow 0
$$

A simple mixing argument which uses (4.1) yields

$$
\sum_{t=1}^{m} \epsilon_{\left(Z_{t}(n) / a(m), \mathbf{X}_{t-1, q}(n)\right)} \Rightarrow \sum_{k} \epsilon_{\left(j_{k}, \mathbf{Y}_{k, q}\right)}
$$

where $\left\{\mathbf{Y}_{k, q}\right\}$ are iid, independent of $\left\{j_{k}\right\}$ and

$$
\mathbf{Y}_{1, q} \stackrel{d}{=} \sum_{j=0}^{q} \mathbf{c}_{j} Z_{j}
$$


with $c_{j}=\lim _{n \rightarrow \infty} c_{j}(n)$ (viz. Proposition 3.4). This mixing argument is standard and can be based, for example, on Theorem 2.1 in Davis and Resnick (1988). See also Davis and McCormick (1989) and Datta and McCormick (1995).

Since

$$
\sum_{k} \epsilon_{\left(j_{k}, \mathbf{Y}_{k, q}\right)} \Rightarrow \sum_{k} \epsilon_{\left(j_{k}, \mathbf{Y}_{k}\right)}
$$

as $q \rightarrow \infty$, in order to show (4.4) it suffices to show

$$
\lim _{q \rightarrow \infty} \limsup _{n \rightarrow \infty} P\left[\rho\left(\sum_{t=1}^{m} \epsilon_{\left(Z_{t}(n) / a(m), \mathbf{X}_{t-1, q}(n)\right)}, \sum_{t=1}^{m} \epsilon_{\left(Z_{t}(n) / a(m), \mathbf{X}_{t-1}(n)\right)}\right)>\eta\right]=0
$$

where $\rho$ is the vague metric on $M_{p}\left([0, \infty) \times([-\infty, \infty] \backslash\{0\})^{p}\right)$ To show (4.10) it suffices to show for any $f \in C_{K}^{+}\left([0, \infty) \times([-\infty, \infty] \backslash\{0\})^{p}\right)$ that

$$
\lim _{q \rightarrow \infty} \limsup _{n \rightarrow \infty} P\left[\left|\sum_{t=1}^{m} f\left(Z_{t}(n) / a(m), \mathbf{X}_{t-1, q}(n)\right)-\sum_{t=1}^{m} f\left(Z_{t}(n) / a(m), \mathbf{X}_{t-1}(n)\right)\right|>\eta\right]=0 .
$$

Because $f$ has compact support, there exists some $c$ such that $f(z, \mathbf{x})$ is 0 if $z>c$. So we write

$$
\begin{aligned}
\sum_{t=1}^{m}\left|f\left(Z_{t}(n) / a(m), \mathbf{X}_{t-1, q}(n)\right)-f\left(Z_{t}(n) / a(m), \mathbf{X}_{t-1}(n)\right)\right| \\
=\sum_{t=1}^{m}\left|f\left(Z_{t}(n) / a(m), \mathbf{X}_{t-1, q}(n)\right)-f\left(Z_{t}(n) / a(m), \mathbf{X}_{t-1}(n)\right)\right| 1_{\left[Z_{t}(n) / a(m)>c\right]} \\
\quad+\sum_{t=1}^{m}\left|f\left(Z_{t}(n) / a(m), \mathbf{X}_{t-1, q}(n)\right)-f\left(Z_{t}(n) / a(m), \mathbf{X}_{t-1}(n)\right)\right| 1_{\left[Z_{t}(n) / a(m) \leq c,\left\|\mathbf{X}_{t-1, q}(n)-\mathbf{X}_{t-1}(n)\right\|>\delta\right]} \\
\quad+\sum_{t=1}^{m}\left|f\left(Z_{t}(n) / a(m), \mathbf{X}_{t-1, q}(n)\right)-f\left(Z_{t}(n) / a(m), \mathbf{X}_{t-1}(n)\right)\right| 1_{\left[Z_{t}(n) / a(m) \leq c,\left\|\mathbf{X}_{t-1, q}(n)-\mathbf{X}_{t-1}(n)\right\| \leq \delta\right]} \\
=A+B+C .
\end{aligned}
$$

Note $A=0$. For $C$ define the modulus of continuity of $f$ to be

$$
\omega_{\delta}(f)=\sup \{|f(\mathbf{x})-f(\mathbf{y})|:\|\mathbf{x}-\mathbf{y}\| \leq \delta\}
$$

and we have by Chebyshev

$$
E(C) \leq \omega_{\delta}(f) m P\left[Z_{1}(n) / a(m) \leq c\right]
$$

and applying (4.2), the right side is asymptotic to

$$
\sim \omega_{\delta}(f) c^{\alpha}
$$

which can be made as small as desired by choosing $\delta$ small. To analyze $B$ we proceed as follows. 


$$
\begin{aligned}
E(B) & \leq 2\|f\| P\left[Z_{1}(n) / a(m) \leq c\right] \sum_{t=1}^{m} P\left[\left\|\mathbf{X}_{t-1, q}(n)-\mathbf{X}_{t-1}(n)\right\|>\delta\right] \\
& \leq 2\|f\| m P\left[Z_{1}(n) / a(m) \leq c\right]\left(\frac{q}{m}+\frac{1}{m} \sum_{t=q+1}^{m} P\left[\left\|\mathbf{X}_{t-1, q}(n)-\mathbf{X}_{t-1}(n)\right\|>\delta\right]\right) \\
& \leq 4\|f\| c^{\alpha}\left(\frac{q}{m}+\frac{1}{m} \sum_{t=q+1}^{m} P\left[\left\|\mathbf{X}_{t-1, q}(n)-\mathbf{X}_{t-1}(n)\right\|>\delta\right]\right)
\end{aligned}
$$

and from (4.7) this has a Chebyshev bound of

$$
\leq 4\|f\| c^{\alpha}\left(\frac{q}{m}+\max _{q<t \leq m} E\left\|\mathbf{X}_{t, q}(n)-\mathbf{X}_{t}(n)\right\|^{\beta} \delta^{-\beta}\right)
$$

Thus we have, again using (4.7), that

$$
\begin{aligned}
\lim _{q \rightarrow \infty} \limsup _{n \rightarrow \infty} P & {\left[\left|\sum_{t=1}^{m} f\left(Z_{n}(n) / a(m), \mathbf{X}_{t-1, q}(n)\right)-\sum_{t=1}^{m} f\left(Z_{n}(n) / a(m), \mathbf{X}_{t-1}(n)\right)\right|>\eta\right] } \\
& \leq(\eta / 3)^{-1} \lim _{q \rightarrow \infty} \limsup _{n \rightarrow \infty}(E(C)+E(B)) \\
& \leq(\eta / 3)^{-1}\left(c^{\alpha} \omega_{\delta}(f)\right. \\
& =(\eta / 3)^{-1}\left(c^{\alpha} \omega_{\delta}(f)\right.
\end{aligned}
$$

and since $\delta$ can be made arbitrarily small, this completes the proof of (4.4).

We now turn to the proof of (4.6). We hope to obtain (4.6) from (4.4) by applying Proposition 3.18, page 148 of Resnick (1987) but the compactness condition (3.15) on page 148 fails and so a truncation is necessary. In (4.4) we truncate the state space to a compact region and so define, for any $M>0$, $S_{M}=\left\{(z, \mathbf{x}): z \leq M,\|\mathbf{x}\|>M^{-1}\right\}$. Then

$$
\lambda_{m}^{M}=\lambda_{m}\left(F_{n}, \phi(n)\right)\left(S_{M} \cap \cdot\right) \Rightarrow \lambda_{\infty}\left(S_{M} \cap \cdot\right)=\lambda_{\infty}^{M}
$$

and on the truncated space we may apply the division map $T$ to obtain $T \lambda_{m}^{M} \Rightarrow T \lambda_{\infty}^{M}$.

We now have to remove the truncation and (4.6) will follow if for any $f \in C_{K}^{+}\left(([-\infty, \infty] \backslash\{0\})^{p}\right)$ we have

$$
\lim _{M \rightarrow \infty} \limsup _{n \rightarrow \infty} P\left[\left|\sum_{t=1}^{m} f\left(\frac{a(m)}{Z_{t}(n)} \mathbf{X}_{t-1}(n)\right) 1_{\left[Z_{t}(n) / a(m) \leq M,\left\|\mathbf{X}_{t-1}(n)\right\|>M^{-1}\right]}-f\left(\frac{a(m)}{Z_{t}(n)} \mathbf{X}_{t-1}(n)\right)\right|>\delta\right]=0 .
$$

The complement of the indicated event in (4.12) is

$$
\left\{\frac{Z_{t}(n)}{a(m)}>M\right\} \bigcup\left\{\left\|\mathbf{X}_{t-1}(n)\right\| \leq M^{-1}\right\}=\left\{\frac{Z_{t}(n)}{a(m)}>M\right\} \bigcup\left\{\frac{Z_{t}(n)}{a(m)} \leq M,\left\|\mathbf{X}_{t-1}(n)\right\| \leq M^{-1}\right\} .
$$


If we suppose the compact support of $f$ is contained in $\{\mathbf{x}:\|\mathbf{x}\|>c\}$ then the probability in (4.12) is bounded by

$$
\begin{aligned}
P\left[\bigcup _ { t = 1 } ^ { m } \left\{\frac{Z_{t}(n)}{a(m)} \leq\right.\right. & \left.\left.M,\left\|\mathbf{X}_{t-1}(n)\right\| \leq M^{-1}, \frac{\left\|\mathbf{X}_{t-1}(n)\right\|}{Z_{t}(n) / a(m)}>c\right\}\right] \\
& +P\left[\bigcup_{t=1}^{m}\left\{\frac{Z_{t}(n)}{a(m)}>M, \frac{\left\|\mathbf{X}_{t-i}(n)\right\|}{Z_{t}(n) / a(m)}>c\right\}\right] \\
= & A+B .
\end{aligned}
$$

Now

$$
A \leq m P\left[\frac{Z_{t}(n)}{a(m)} \leq(c M)^{-1}\right] \sim(c M)^{-\alpha}
$$

which goes to zero as $M \rightarrow \infty$. For $B$ we have

$$
\begin{aligned}
B & \leq \sum_{t=1}^{m} P\left[\frac{Z_{t}(n)}{a(m)}>M, \frac{\left\|\mathbf{X}_{t-1}(n)\right\|}{Z_{t}(n) / a(m)}>c\right] \\
& \leq \sum_{t=1}^{m} P\left[\frac{\left\|\mathbf{X}_{t-1}(n)\right\|}{Z_{t}(n) / a(m)} 1_{\left[\left(Z_{t}(n) / a(m)\right)^{-1} \leq M^{-1}\right]}>c\right] \\
& \leq c^{-\beta} \sum_{t=1}^{m} E\left(\left\|\mathbf{X}_{t-1}(n)\right\|^{\beta}\right) E\left[\left(Z_{t}(n) / a(m)\right)^{-\beta} 1_{\left[\left(Z_{t}(n) / a(m)\right)^{-1} \leq M^{-1}\right]}\right] .
\end{aligned}
$$

Using (4.7) again this last expression is bounded by

$$
\leq(\text { const }) m E\left(\left(Z_{1}(n) / a(m)\right)^{-1}\right)^{\beta} 1_{\left[\left(Z_{1}(n) / a(m)\right)^{-1} \leq M^{-1}\right]}
$$

and an appeal to condition (4.5) finishes the proof.

For consideration of the right tail case, we will need the following extension of a result of Cline (1983) which relates tail behavior of the distribution of a linear combination of iid random variables to tail behavior of one of the summands.

Proposition 4.2. Assume we have non-negative constants $\left\{\psi_{j}(n) ; j \geq 0, n \geq 0\right\}$ such that:

$$
\lim _{n \rightarrow \infty} \psi_{j}(n)=\psi_{j}(0) ; j \geq 0
$$

and for all $0<\delta$

$$
\sum_{j=0}^{\infty} \psi_{j}(n)^{\delta}<\infty, n \geq 0 ; \lim _{q \rightarrow \infty} \limsup _{n \rightarrow \infty} \sum_{j=q}^{\infty} \psi_{j}(n)^{\delta}=0 .
$$

Let $\left\{Z_{t}(n), t \geq 1\right\}$ be iid with common distribution function $F_{n}$ satisfying (as $n \rightarrow \infty$ )

$$
\begin{gathered}
m\left(1-F_{n}(b(m) x)\right) \rightarrow x^{-\alpha}, \quad x>0, \\
\infty>\sum_{j=0}^{\infty} m\left(1-F_{n}\left(b(m) \psi_{j}(n)^{-1} x\right)\right) \rightarrow \sum_{j=0}^{\infty} \psi_{j}(0)^{\alpha} x^{-\alpha}, \quad x>0,
\end{gathered}
$$




$$
\infty>\sum_{j=0}^{\infty} \int_{0}^{x} u^{\beta-1} m\left(1-F_{n}\left(b(m) \psi_{j}(n)^{-1} u\right)\right) d u \rightarrow \sum_{j=0}^{\infty} \psi_{j}(0)^{\alpha} \frac{x^{\beta-\alpha}}{\beta-\alpha}, x>0, \text { for } \beta>\alpha .
$$

Then as $n \rightarrow \infty$ we have

$$
m P\left[\sum_{j=0}^{\infty} \psi_{j}(n) Z_{j}(n)>b(m) x\right] \rightarrow \sum_{j=0}^{\infty} \psi_{j}(0)^{\alpha} x^{-\alpha} \quad x>0 .
$$

In addition, suppose $\{\zeta(n), n \geq 0\}$ are non-negative random variables satisfying $\zeta(n) \Rightarrow \zeta(0)$ as $n \rightarrow \infty$, $\zeta(n)$ is independent of $\left\{Z_{t}(n), t \geq 1\right\}$ and for some $\beta>\alpha$

$$
\sup _{n \geq 0} E \zeta(n)^{\beta}<\infty .
$$

Then

$$
m P\left[\zeta(n) \sum_{j=0}^{\infty} \psi_{j}(n) Z_{j}(n)>b(m) x\right] \rightarrow \sum_{j=0}^{\infty} \psi_{j}(0)^{\alpha} x^{-\alpha} E \zeta(0)^{\alpha}
$$

Proof. Let $\left\{Z_{t}(n)^{\prime}, Z_{t}(n)^{\prime \prime}, t \geq 1\right\}$ be iid copies of $\left(Z_{1}(n), Z_{2}(n)\right)$. It is readily checked that in $[0, \infty]^{2} \backslash\{\mathbf{0}\}$ we have

$m P\left[\left(\psi_{1}(n) \frac{Z_{t}(n)^{\prime}}{b(m)}, \psi_{2}(n) \frac{Z_{t}(n)^{\prime \prime}}{b(m)}\right) \in \cdot\right] \stackrel{v}{\rightarrow} \psi_{1}(0)^{\alpha} \nu\left(d z_{1}\right) \times \epsilon_{0}\left(d z_{2}\right)+\epsilon_{0}\left(d z_{1}\right) \times \psi_{2}(0)^{\alpha} \nu\left(d z_{2}\right)=: \nu^{\#}\left(d z_{1}, d z_{2}\right)$

where as usual $\nu(d z)=\alpha z^{-\alpha-1} d z, z>0$. Thus from Proposition 3.21 in Resnick (1987) we conclude

$$
\sum_{t=1}^{m} \epsilon_{\left(\psi_{1}(n) \frac{Z_{t}(n)^{\prime}}{b(m)}, \psi_{2}(n) \frac{\left.Z_{t}(n)^{\prime \prime}\right)}{b(m)}\right)} \Rightarrow \sum_{k} \epsilon_{\left(\psi_{1}(0) j_{k}^{\prime}, 0\right)}+\sum_{k} \epsilon_{\left(0, \psi_{2}(0) j_{k}^{\prime \prime}\right)}
$$

where the Poisson limit is the sum of two independent Poisson processes and has mean measure $\nu^{\#}$. Applying the summation functional to the points and using Proposition 3.18 in Resnick (1987) we get

$$
\sum_{t=1}^{m} \epsilon_{\left(\psi_{1}(n) Z_{t}(n)^{\prime}+\psi_{2}(n) Z_{t}(n)^{\prime \prime}\right) / b(m)} \Rightarrow \sum_{k} \epsilon_{\left(\psi_{1}(0) j_{k}^{\prime}\right)}+\sum_{k} \epsilon_{\left(\psi_{2}(0) j_{k}^{\prime \prime}\right)}
$$

where the limit is again the sum of two independent Poisson processes. Again applying Proposition 3.21 in Resnick (1987) we conclude that as $n \rightarrow \infty$

$$
\begin{aligned}
m P\left[\psi_{1}(n) Z_{1}(n)^{\prime}\right. & \left.+\psi_{2}(n) Z_{1}(n)^{\prime \prime}>b(m) x\right] \\
& \rightarrow E\left(\sum_{k} \epsilon_{\left(\psi_{1}(0) j_{k}^{\prime}\right)}+\sum_{k} \epsilon_{\left(\psi_{2}(0) j_{k}^{\prime \prime}\right)}\right)(x, \infty] \\
& =\left(\psi_{1}(0)^{\alpha}+\psi_{2}(0)^{\alpha}\right) x^{-\alpha}
\end{aligned}
$$

This result can be extended to any finite sum so for any integer $q$ we conclude

$$
m P\left[\sum_{t=0}^{q} \psi_{t}(n) Z_{t}(n)>b(m) x\right] \rightarrow \sum_{i=0}^{q} \psi_{i}(0)^{\alpha} x^{-\alpha}, \quad x>0 .
$$


Now write

$$
\begin{gathered}
m P\left[\sum_{j=0}^{\infty} \psi_{j}(n) Z_{j}(n)>b(m) x\right] \\
=m P\left[\sum_{j=0}^{\infty} \psi_{j}(n) Z_{j}(n)>b(m) x, \bigvee_{j=0}^{\infty} \psi_{j}(n) Z_{j}(n)>b(m) x\right] \\
\quad+m P\left[\sum_{j=0}^{\infty} \psi_{j}(n) Z_{j}(n)>b(m) x, \bigvee_{j=0}^{\infty} \psi_{j}(n) Z_{j}(n) \leq b(m) x\right] \\
\leq \sum_{j=0}^{\infty} m P\left[\psi_{j}(n) Z_{j}(n)>b(m) x\right] \\
\quad+m P\left[\sum_{j=0}^{\infty} \psi_{j}(n) \frac{Z_{j}(n)}{b(m)} 1_{\left[\psi_{j}(n) \frac{Z_{j}(n)}{b(m)} \leq x\right]}>x\right] \\
=I+I I .
\end{gathered}
$$

We have

$$
I \rightarrow \sum_{j=0}^{\infty} \psi_{j}(0)^{\alpha} x^{-\alpha}
$$

by assumption (4.14). To control $I I$ we assume $\alpha<\beta \leq 1$ with a similar argument applying if $\beta>1$ (see $2.20,4.7)$. Then we have

$$
\begin{aligned}
I I & =m P\left[\left(\sum_{j=0}^{\infty} \psi_{j}(n) \frac{Z_{j}(n)}{b(m)} 1_{\left[\psi_{j}(n) \frac{Z_{j}(n)}{b(m)} \leq x\right]}\right)^{\beta}>x^{\beta}\right] \\
& \leq x^{-\beta} \sum_{j=0}^{\infty} m E\left[\left(\frac{\psi_{j}(n) Z_{j}(n)}{b(m)}\right)^{\beta} 1_{\left[\frac{\psi_{j}(n) Z_{j}(n)}{b(m)} \leq x\right]}\right] \\
& \leq x^{-\beta} \sum_{j=0}^{\infty} \int_{0}^{x} \beta u^{\beta-1} m P\left[\frac{\psi_{j}(n) Z_{j}(n)}{b(m)}>u\right] d u \\
& \rightarrow x^{-\beta} \sum_{j=0}^{\infty} \psi_{j}(0)^{\alpha} \frac{\beta x^{\beta-\alpha}}{\beta-\alpha} \quad(\text { from (4.15)) } \\
& =\sum_{j=0}^{\infty} \psi_{j}(0)^{\alpha} \frac{\beta x^{-\alpha}}{\beta-\alpha} .
\end{aligned}
$$

Thus, on the one hand, for any $q$, as $n \rightarrow \infty$

$$
m P\left[\sum_{j=0}^{\infty} \psi_{j}(n) \frac{Z_{j}(n)}{b(m)}>x\right] \geq m P\left[\sum_{j=0}^{q} \psi_{j}(n) \frac{Z_{j}(n)}{b(m)}>x\right] \rightarrow \sum_{j=0}^{q} \psi_{j}(0)^{\alpha} x^{-\alpha}
$$


and so letting $q \rightarrow \infty$,

$$
\liminf _{n \rightarrow \infty} m P\left[\sum_{j=0}^{\infty} \psi_{j}(n) \frac{Z_{j}(n)}{b(m)}>x\right] \geq \sum_{j=0}^{\infty} \psi_{j}(0)^{\alpha} x^{-\alpha} .
$$

On the other hand, for any small $\delta$ and any $q$

$$
\begin{aligned}
& m P\left[\sum_{j=0}^{\infty} \psi_{j}(n) \frac{Z_{j}(n)}{b(m)}>x\right] \\
& \quad \leq m P\left[\sum_{j=0}^{q} \psi_{j}(n) \frac{Z_{j}(n)}{b(m)}>x(1-\delta)\right]+m P\left[\sum_{j=q+1}^{\infty} \psi_{j}(n) \frac{Z_{j}(n)}{b(m)}>x \delta\right] \\
& \quad=A+B .
\end{aligned}
$$

Now from (4.19) we get

$$
A \rightarrow \sum_{j=0}^{q} \psi_{j}(0)^{\alpha}(x(1-\delta))^{-\alpha}
$$

while from (4.20) and (4.21)

$$
\limsup _{n \rightarrow \infty} B \leq \sum_{j=q+1}^{\infty} \psi_{j}(0)^{\alpha}\left(\frac{\beta}{\beta-\alpha}+1\right)(x \delta)^{-\alpha} .
$$

Thus

$$
\begin{aligned}
\limsup _{n \rightarrow \infty} & m P\left[\sum_{j=0}^{\infty} \psi_{j}(n) \frac{Z_{j}(n)}{b(m)}>x\right] \\
\leq & \sum_{j=0}^{q} \psi_{j}(0)^{\alpha}(x(1-\delta))^{-\alpha}+\left(\frac{\beta}{\beta-\alpha}+1\right) \sum_{j=q+1}^{\infty} \psi_{j}(0)^{\alpha}(x \delta)^{-\alpha} .
\end{aligned}
$$

On the right side, let $q \rightarrow \infty$ and then let $\delta \downarrow 0$ to obtain the desired result.

In order to focus on the proof of (4.18) we set

$$
X^{\prime}(n)=\sum_{j=0}^{\infty} \psi_{j}(n) Z_{j}(n)
$$

We claim that for proving (4.18), it is enough to show

$$
\lim _{M \rightarrow \infty} \limsup _{n \rightarrow \infty}\left|m P\left[\zeta(n) X^{\prime}(n)>b(m) \delta\right]-m P\left[\zeta(n) X^{\prime}(n)>b(m) \delta, \zeta(n) \leq M\right]\right|=0 .
$$

To see this, note that

$$
\begin{array}{r}
\left|\int_{0}^{M} m P\left[X^{\prime}(n)>b(m) u^{-1} \delta\right] P[\zeta(n) \in d u]-\int_{0}^{M} \sum_{j=0}^{\infty} \psi_{j}(0)^{\alpha} \delta^{-\alpha} u^{\alpha} P[\zeta(n) \in d u]\right| \\
\leq \int_{0}^{M}\left|m P\left[X^{\prime}(n)>b(m) u^{-1} \delta\right]-\sum_{j=0}^{\infty} \psi_{j}(0)^{\alpha} \delta^{-\alpha} u^{\alpha}\right| P[\zeta(n) \in d u] .
\end{array}
$$


Now the integrand converges to 0 uniformly on $u$-neighborhoods of 0 and so for any $\epsilon$, we have for $n$ sufficiently large that the above difference is bounded by $\epsilon P[\zeta(n) \leq M] \leq \epsilon$ and so we conclude

$$
\int_{0}^{M} m P\left[X^{\prime}(n)>b(m) u^{-1} \delta\right] P[\zeta(n) \in d u] \rightarrow \int_{0}^{M} \sum_{j=0}^{\infty} \psi_{j}(0)^{\alpha} \delta^{-\alpha} u^{\alpha} P[\zeta(0) \in d u] .
$$

So if (4.22) is true then

$$
\begin{aligned}
&\left|m P\left[\zeta(n) X^{\prime}(n)>b(m) \delta\right]-\delta^{-\alpha} \sum_{j=0}^{\infty} \psi_{j}(0)^{\alpha} \int_{0}^{\infty} u^{\alpha} P[\zeta(0) \in d u]\right| \\
& \leq\left|m P\left[\zeta(n) X^{\prime}(n)>b(m) \delta\right]-m P\left[\zeta(n) X^{\prime}(n)>b(m) \delta, \zeta(n) \leq M\right]\right| \\
&+\left|m P\left[\zeta(n) X^{\prime}(n)>b(m) \delta, \zeta(n) \leq M\right]-\delta^{-\alpha} \sum_{j=0}^{\infty} \psi_{j}(0)^{\alpha} \int_{0}^{M} u^{\alpha} P[\zeta(0) \in d u]\right| \\
&+\left|\delta^{-\alpha} \sum_{j=0}^{\infty} \psi_{j}(0)^{\alpha} \int_{0}^{M} u^{\alpha} P[\zeta(0) \in d u]-\delta^{-\alpha} \sum_{j=0}^{\infty} \psi_{j}(0)^{\alpha} \int_{0}^{\infty} u^{\alpha} P[\zeta(0) \in d u]\right| \\
&=A+B+C .
\end{aligned}
$$

Now $C \rightarrow 0$ as $M \rightarrow \infty$ since $E \zeta(0)^{\alpha}<\infty$. Also $B \rightarrow 0$ from (4.23) and $\lim _{M \rightarrow \infty} \limsup _{n \rightarrow \infty} A=0$ if $(4.22)$ is true. So it remains to prove (4.22).

The difference in the probabilities in (4.22) is

$$
\begin{aligned}
m P\left[\zeta(n) X^{\prime}(n)>\right. & b(m) \delta, \zeta(n)>M] \\
= & m P\left[\zeta(n) X^{\prime}(n)>b(m) \delta, \zeta(n)>M, \bigvee_{j=0}^{\infty} \psi_{j}(n) Z_{j}(n)>M^{-1} b(m)\right] \\
& \quad+m P\left[\zeta(n) X^{\prime}(n)>b(m) \delta, \zeta(n)>M, \bigvee_{j=0}^{\infty} \psi_{j}(n) Z_{j}(n) \leq M^{-1} b(m)\right] \\
& =A+B .
\end{aligned}
$$

For $A$ we have

$$
\begin{aligned}
A & \leq P[\zeta(n)>M] m \sum_{j=0}^{\infty}\left(1-F_{n}\left(\psi_{j}(n)^{-1} M^{-1} b(m)\right)\right) \\
& \rightarrow P[\zeta(0)>M] \sum_{j=0}^{\infty} \psi_{j}(0)^{\alpha} M^{\alpha} \quad(n \rightarrow \infty) \\
& \rightarrow 0, \quad(M \rightarrow \infty),
\end{aligned}
$$

since $E \zeta(0)^{\beta}<\infty$. For $B$ the calculations look like this:

$$
B \leq m P\left[\zeta(n) \sum_{j=0}^{\infty} \psi_{j}(n) \frac{Z_{j}(n)}{b(m)} 1_{\left[\psi_{j}(n) \frac{Z_{j}(n)}{b(m)} \leq M^{-1}\right]}>\delta, \zeta(n)>M\right]
$$

and supposing $0<\alpha<\beta \leq 1$ with a similar argument in the contrary case, we get a bound of 


$$
\begin{aligned}
& \leq m P\left[\zeta(n)^{\beta} \sum_{j=0}^{\infty} \psi_{j}(n)^{\beta}\left(\frac{Z_{j}(n)}{b(m)}\right)^{\beta} 1_{\left[\psi_{j}(n) \frac{Z_{j}(n)}{b(m)} \leq M^{-1}\right]}>\delta^{\beta}\right] \\
& \leq \delta^{-\beta} m E \zeta(n)^{\beta} \sum_{j=0}^{\infty} E\left[\left(\psi_{j}(n)\left(\frac{Z_{j}(n)}{b(m)}\right)\right)^{\beta} 1_{\left[\psi_{j}(n) \frac{Z_{j}(n)}{b(m)} \leq M^{-1}\right]}\right] \\
& \leq \delta^{-\beta} E \zeta(n)^{\beta} m \sum_{j=0}^{\infty} \int_{0}^{M^{-1}} \beta u^{\beta-1} P\left[\psi_{j}(n) \frac{Z_{j}(n)}{b(m)}>u\right] d u .
\end{aligned}
$$

Then using assumptions (4.15) and (4.17) we have

$$
\limsup _{n \rightarrow \infty} B \leq\left(\sup _{n \geq 0} E \zeta(n)^{\beta}\right) \delta^{-\beta} \sum_{j=0}^{\infty} \psi_{j}(0)^{\alpha} \frac{\left(M^{-1}\right)^{\beta-\alpha}}{\beta-\alpha} \rightarrow 0
$$

as $M \rightarrow \infty$. This completes the proof.

We now consider the triangular array limit theorem necessary for bootstrapping the right tail case.

Theorem 4.3. Suppose $\left\{Z_{t}\right\}$ iid $F$, and $\left\{X_{t}\right\}$ is the autoregression of (1.1) such that $F$ and $\phi$ satisfy conditions $M, S$ and $R$ with $b(n)=F^{\leftarrow}\left(1-\frac{1}{n}\right)$. For each $n$, suppose $\left\{Z_{t}(n), t \geq 1\right\}$ is iid with common distribution $F_{n}$. Suppose $Z_{t}(n)=0, t \leq 0$. Parallel to (4.1)-(4.3) we assume

(1) $F_{n}$ approximates $F$ weakly:

$$
F_{n} \Rightarrow F \quad(n \rightarrow \infty),
$$

(2) $F_{n}$ approximates $\nu(d x):=\alpha x^{-\alpha-1} d x 1_{(0, \infty]}$ vaguely:

$$
m F_{n}(b(m) \cdot) \stackrel{v}{\rightarrow} \nu, \quad(n \rightarrow \infty)
$$

in $M_{+}((0, \infty])$ where $m=m(n) \rightarrow \infty$.

In addition, assume (4.14) and (4.15) hold.

Suppose we have vectors $\phi(n) \in \mathbb{R}^{p}$ such that $\phi(n) \rightarrow \phi$. Define $\Phi_{n}(z), C_{n}(z)$ as in Proposition 3.4, and $\left\{Z_{t}(n)\right\},\left\{X_{t}(n)\right\}$ and $\left\{\mathbf{X}_{t}(n)\right\}$ as in $(2.3-2.5)$.

Then in $M_{p}((0, \infty])$ as $n \rightarrow \infty$

$$
\mu_{m}\left(F_{n}\right)=\sum_{i=1}^{m} \epsilon_{b(m)^{-1} Z_{t}(n)} \Rightarrow \sum_{k} \epsilon_{j_{k}}
$$

where the limit is $\operatorname{PRM}(\nu)$ with $\nu(x, \infty]=x^{-\alpha}$. Also, as $n \rightarrow \infty$

$$
\lambda_{m}\left(F_{n}, \phi(n)\right)=\sum_{t=1}^{m} \epsilon_{\left(Z_{t}(n), b(m)^{-1} \mathbf{X}_{t-1}(n)\right)} \Rightarrow \sum_{k} \sum_{l=0}^{\infty} \epsilon_{\left(Y_{k l}, j_{k} \mathbf{c}_{l}\right)}
$$

in $M_{p}\left([0, \infty) \times\left([-\infty, \infty]^{p} \backslash\{\mathbf{0}\}\right)\right)$ where $\left\{Y_{k l}\right\}$ are iid, independent of $\left\{j_{k}\right\}$ and have the same distribution as $Z_{1}$.

Finally, if for any $M>0$

$$
\sup _{n \geq 1} E Z_{1}(n)^{-\beta} 1_{\left[Z_{1}(n)^{-1}>M\right]}<\infty
$$


then

$$
\sum_{t=1}^{m} \epsilon_{b(m)^{-1} Z_{t}(n)^{-1} \mathbf{X}_{t-1}(n)} \Rightarrow \sum_{k} \sum_{l=0}^{\infty} \epsilon_{\left(j_{k} / Y_{k l}\right) \mathbf{c}_{l}}
$$

in $M_{p}\left(([-\infty, \infty] \backslash\{0\})^{p}\right)$.

Proof. The proof is similar to Propositions 4.27, Resnick (1987) (which summarizes some of the work in Davis and Resnick (1985)), Theorem 4.3 in Feigin and Resnick (1994), Proposition 3.3 in Feigin and Resnick (1992) and we only outline some parts of this proof.

We begin by observing that (4.25) is equivalent to

$$
\sum_{t=1}^{m} \epsilon_{b(m)^{-1} Z_{t}(n)} \Rightarrow \sum_{k} \epsilon_{j_{k}}
$$

in $M_{p}((0, \infty])$ by Proposition 3.21, Resnick (1987), and for any fixed integer $q$ this leads to the following convergence in $M_{p}\left([0, \infty) \times\left([0, \infty]^{q} \backslash\{\mathbf{0}\}\right)\right)$ :

$$
\begin{aligned}
I_{n}:= & \sum_{t=1}^{m} \epsilon_{\left(Z_{t}(n),\left(Z_{t-1}(n) / b(m), \ldots, Z_{t-q}(n) / b(m)\right)\right)} \\
& \Rightarrow \sum_{k} \sum_{l=1}^{q} \epsilon_{\left(Y_{k l}, j_{k} \mathbf{e}_{l}\right)}=: I_{\infty},
\end{aligned}
$$

where $\mathbf{e}_{1}=(1, \ldots, 0), \ldots, \mathbf{e}_{q}=(0, \ldots, 1)$. To verify this claimed convergence of $I_{n} \Rightarrow I_{\infty}$ let $\rho$ be the vague metric on $M_{p}\left([0, \infty) \times\left([0, \infty]^{q} \backslash\{\mathbf{0}\}\right)\right)$ and set

$$
\begin{aligned}
I_{n}^{*} & =\sum_{t=1}^{m} \sum_{l=1}^{q} \epsilon_{\left(Z_{t}(n), b(m)^{-1} Z_{t-l}(n) \mathbf{e}_{l}\right)}, \\
I_{n}^{* *} & =\sum_{t=1}^{m} \sum_{l=1}^{q} \epsilon_{\left(Z_{t+l}(n), b(m)^{-1} Z_{t}(n) \mathbf{e}_{l}\right)},
\end{aligned}
$$

and then we have

$$
\rho\left(I_{n}, I_{n}^{*}\right) \stackrel{P}{\rightarrow} 0, \quad \rho\left(I_{n}^{*}, I_{n}^{* *}\right) \stackrel{P}{\rightarrow} 0
$$

and so it suffices to check $I_{n}^{* *} \Rightarrow I_{\infty}$.

Observe that

$$
\sum_{t=1}^{m} \epsilon_{\left(Z_{t+1}(n), \ldots, Z_{t+q}(n), b(m)^{-1} Z_{t}(n)\right)} \Rightarrow \sum_{k} \epsilon_{\left(Y_{k 1}, \ldots, Y_{k q}, j_{k}\right)}
$$

in $M_{p}\left([0, \infty)^{q} \times(0, \infty]\right)$, where $\left\{Y_{k j}, k \geq 1, j \geq 1\right\}$ are iid with common distribution $F$. To verify (4.29), set

$$
\mathbf{W}_{n, t}=\left(Z_{t+1}(n), \ldots, Z_{t+q}(n), \frac{Z_{t}(n)}{b(m)}\right)
$$

and note from (4.24) and (4.25) that

$$
m P\left[\mathbf{W}_{n, 1} \in\left(d z_{1}, \ldots, d z_{q}, d z_{0}\right)\right] \stackrel{v}{\rightarrow} \nu\left(d z_{0}\right) \prod_{i=1}^{q} F\left(d z_{i}\right) .
$$


Also, let $g \in C_{K}^{+}\left([0, \infty)^{q} \times(0, \infty]\right)$ and suppose the support of $g$ is such that $g\left(z_{1}, \ldots, z_{q}, z_{0}\right)=0$ if $z_{0}<\delta$. Then

$$
\begin{aligned}
\lim _{k \rightarrow \infty} \limsup _{n \rightarrow \infty} m \sum_{i=2}^{[m / k]} E\left(g\left(\mathbf{W}_{n, 1}\right) g\left(\mathbf{W}_{n, i}\right)\right) \\
\leq \lim _{k \rightarrow \infty} \limsup _{n \rightarrow \infty} m \sum_{i=2}^{[m / k]} P\left[\frac{Z_{1}(n)}{b(m)}>\delta, \frac{Z_{i}(n)}{b(m)}>\delta\right] \\
\leq \lim _{k \rightarrow \infty} \limsup _{n \rightarrow \infty} m \frac{m}{k}\left(1-F_{n}(\delta b(m))\right)^{2} \\
=\lim _{k \rightarrow \infty} \frac{\delta^{-2 \alpha}}{k} \quad(\text { from }(4.25)) \\
=0
\end{aligned}
$$

and so Theorem 2.1 in Davis and Resnick (1988) assures us that (4.29) holds.

The continuous mapping theorem then guarantees that (4.29) implies, basically by transforming $\left(z_{1}, \ldots, z_{q}, x\right) \mapsto\left(z_{l}, x \mathbf{e}_{l}\right)$ and then adding over $l$, that

$$
I_{n}^{* *}=\sum_{l=1}^{q} \sum_{t=1}^{m} \epsilon_{\left(Z_{t+l}(n), b(m)^{-1} Z_{t}(n) \mathbf{e}_{l}\right)} \Rightarrow \sum_{l=1}^{q} \sum_{k} \epsilon_{\left.Y_{k l}, j_{k} \mathbf{e}_{l}\right)}:=I_{\infty}
$$

in $M_{p}\left([0, \infty) \times\left([0, \infty]^{q} \backslash\{0\}\right)\right)$. Thus the same holds true for $\left\{I_{n}\right\}$.

Now, recalling the definition of $C_{n}(z)$ and the vector $\mathbf{c}_{l}(n)$ we apply the map

$$
\left.\left(z, x_{1}, \ldots, x_{q}\right)\right) \mapsto\left(z, x_{1} \mathbf{c}_{0}(n)+x_{2} \mathbf{c}_{1}(n)+\cdots+x_{q} \mathbf{c}_{q-1}(n)\right)
$$

to the points of $I_{n}$ to obtain (cf page 235, Resnick, 1987)

$$
\sum_{t=1}^{m} \epsilon_{\left(Z_{t}(n), b(m)^{-1} \mathbf{X}_{t-1, q-1}(n)\right)} \Rightarrow \sum_{k} \sum_{l=1}^{q} \epsilon_{\left(Y_{k l}, j_{k} \mathbf{c}_{l-1}\right)}
$$

in $M_{p}\left([0, \infty) \times([-\infty, \infty] \backslash\{0\})^{p}\right)$ and where $\mathbf{X}_{t, q}(n)=\sum_{j=0}^{q} \mathbf{c}_{j}(n) Z_{t-j}$.

It remains to show we can replace $q$ by $\infty$. (Recall $Z_{t}(n)=0$ if $t \leq 0$.). We have as $q \rightarrow \infty$

$$
\sum_{k} \sum_{l=1}^{q} \epsilon_{\left(Y_{k l}, j_{k} \mathbf{c}_{l-1}\right)} \Rightarrow \sum_{k} \sum_{l=0}^{\infty} \epsilon_{\left(Y_{k l}, j_{k} \mathbf{c}_{l}\right)}
$$

and so by Billingsley, 1968, Theorem 4.2 it suffices to check for any $\eta>0$ that

$$
\operatorname{lims}_{q \rightarrow \infty} \limsup _{n \rightarrow \infty} P\left[\rho\left(\sum_{t=1}^{m} \epsilon_{\left(Z_{t}(n), b(m)^{-1} \mathbf{X}_{t-1, q-1}(n)\right)}, \sum_{t=1}^{m} \epsilon_{\left(Z_{t}(n), b(m)^{-1} \mathbf{X}_{t-1}(n)\right)}\right)>\eta\right]=0,
$$

where $\mathbf{X}_{t}(n)=\sum_{j=0}^{t-1} \mathbf{c}_{j}(n) Z_{t-j}$.

Let $g \in C_{K}^{+}\left([0, \infty) \times([0, \infty) \backslash\{0\})^{p}\right)$ and suppose $g\left(z_{1}, \mathbf{z}_{2}\right)=0$ if $\left\|\mathbf{z}_{2}\right\|<\delta$. We need to show

$$
\begin{aligned}
\lim _{q \rightarrow \infty} \limsup _{n \rightarrow \infty} P\left[\mid \sum_{t=1}^{m} g\left(Z_{t}(n), \sum_{j=0}^{q-1} \mathbf{c}_{j}(n) Z_{t-j}(n) / b(m)\right)-\sum_{t=1}^{m} g\left(Z_{t}(n), \sum_{j=0}^{t-1} \mathbf{c}_{j}(n) Z_{t-j}(n) / b(m)\right)\right. \\
>\eta]=0 .
\end{aligned}
$$


Define the event

$$
[L E S S]=\left[\bigvee_{t=1}^{m} \frac{\left\|\mathbf{X}_{t, q}(n)-\mathbf{X}_{t}(n)\right\|}{b(m)} \leq \theta\right] .
$$

The probability in (4.32) is bounded by

$$
P\left([L E S S]^{c}\right)+P\left[\sum_{t=1}^{m}\left|g\left(Z_{t}(n), \mathbf{X}_{t, q}(n) / b(m)\right)-g\left(Z_{t}(n), \mathbf{X}_{t}(n) / b(m)\right)\right|>\eta, L E S S\right] .
$$

Now

$$
P\left([L E S S]^{c}\right) \leq \sum_{t=1}^{m} P\left[\frac{\left\|\mathbf{X}_{t, q}(n)-\mathbf{X}_{t}(n)\right\|}{b(m)}>\theta\right] \leq m P\left[\sum_{j=q+1}^{\infty}\left\|\mathbf{c}_{j}(n)\right\| Z_{j}(n)>b(m) \theta\right]
$$

and by the extended version of Cline's lemma, Proposition 4.2, and in particular (4.18) we have

$$
\limsup _{n \rightarrow \infty} P\left([L E S S]^{c}\right) \leq \sum_{j=q+1}\left\|\mathbf{c}_{j}\right\|^{\alpha} \theta^{-\alpha} \rightarrow 0 \text { as } q \rightarrow \infty
$$

Let

$$
\omega_{g}(\theta)=\sup \{|g(\mathbf{x})-g(\mathbf{y})|:\|\mathbf{x}-\mathbf{y}\|<\theta\}
$$

and assume $\theta<\delta / 2$. (Recall $g\left(z_{1}, \mathbf{z}_{2}\right)=0$ if $\left\|\mathbf{z}_{2}\right\|<\delta$.) Then the second probability in (4.33) is bounded by

$$
P\left[\omega_{g}(\theta) \sum_{t=1}^{m} \epsilon_{\mathbf{X}_{t, q}(n) / b(m)}\left(\left\{\mathbf{y}:\|\mathbf{y}\|>\frac{1}{2} \delta\right\}\right)>\eta\right]
$$

which from (4.28) converges, as $n \rightarrow \infty$, to

$$
P\left[\omega_{g}(\theta) \sum_{k} \sum_{l=0}^{q-1} \epsilon_{j_{k} \mathbf{c}_{l}}\left(\left\{\mathbf{y}:\|\mathbf{y}\|>\frac{1}{2} \delta\right\}\right)>\eta\right]
$$

and as $q \rightarrow \infty$ we get the limit

$$
P\left[\omega_{g}(\theta) \sum_{k} \sum_{l=0}^{\infty} \epsilon_{j_{k} \mathbf{c}_{l}}\left(\left\{\mathbf{y}:\|\mathbf{y}\|>\frac{1}{2} \delta\right\}\right)>\eta\right] .
$$

Letting $\theta \rightarrow 0$ makes $\omega_{g}(\theta) \rightarrow 0$ and this completes the proof of (4.26).

To prove (4.28), we return to our map $T$ of (2.8) Since $T$ does not have the compactness property discussed in Proposition 3.18 of Resnick (1987), a truncation of the state space is necessary. Choose $M$ large and restricting the state space to $S_{M}=\left\{(z, \mathbf{x}): z \leq M,\|\mathbf{x}\|>M^{-1}\right\}$ in (4.26) yields

$$
\lambda_{m}^{M}=\lambda_{m}\left(F_{n}, \phi(n)\right)\left(S_{M} \cap \cdot\right) \Rightarrow \lambda_{\infty}\left(S_{M} \cap \cdot\right)=\lambda_{\infty}^{M}
$$

and

$$
T \lambda_{m}^{M} \Rightarrow T \lambda_{\infty}^{M}=\sum_{k} \sum_{l=0}^{\infty} 1_{\left[j_{k}\left\|\mathbf{c}_{l-1}\right\|>M^{-1}, Y_{k l} \leq M\right]} \epsilon_{\left(j_{k} Y_{k l}^{-1} \mathbf{c}_{l}\right)} .
$$


Let $M \rightarrow \infty$. The right side approaches the right side of (4.28) and by Billingsley (1968), Theorem 4.2, it suffices to show for any $f \in C_{K}^{+}\left(\left([-\infty, \infty]^{p} \backslash\{0\}\right)\right)$ and $\eta>0$ that

$$
\lim _{M \rightarrow \infty} \limsup _{n \rightarrow \infty} P\left[\sum_{t=1}^{m} f\left(b(m)^{-1} Z_{t}(n)^{-1} \mathbf{X}_{t-1}(n)\right) 1_{\left[\left\|X_{t-1}(n)\right\| / b(m)>M^{-1}, Z_{t}(n) \leq M\right]^{c}}>2 \eta\right]=0 .
$$

This probability is bounded by

$$
\begin{aligned}
& P\left[\sum_{t=1}^{m} f\left(b(m)^{-1} Z_{t}(n)^{-1} \mathbf{X}_{t-1}(n)\right) 1_{\left[Z_{t}(n)>M\right]}>\eta\right] \\
& \quad+P\left[\sum_{t=1}^{m} f\left(b(m)^{-1} Z_{t}(n)^{-1} \mathbf{X}_{t-1}(n)\right) 1_{\left[\left\|\mathbf{X}_{t-1}(n)\right\| / b(m) \leq M^{-1}\right]}>\eta\right] \\
& \quad=A+B .
\end{aligned}
$$

Now suppose the support of $f$ is contained in $\{\mathbf{x}:\|\mathbf{x}\|>\delta\}$. Then

$$
\begin{aligned}
A & \leq P\left[\bigcup_{t=1}^{m}\left\{\frac{\left\|\mathbf{X}_{t-1}(n)\right\|}{b(m) Z_{t}(n)}>\delta, Z_{t}(n)>M\right\}\right] \\
& \leq P\left[\bigcup_{t=1}^{m}\left\{\frac{\left\|\mathbf{X}_{t-1}(n)\right\|}{b(m) M}>\delta\right\}\right] \\
& \leq \sum_{t=1}^{m} P\left[\left\|\mathbf{X}_{t-1}(n)\right\|>b(m) M \delta\right] \\
& \leq m P\left[\sum_{j=0}^{\infty}\left\|\mathbf{c}_{j}(n)\right\| Z_{j}(n)>\delta b(m) M\right] \\
& \rightarrow \sum_{j=0}^{\infty}\left\|\mathbf{c}_{j}(0)\right\|^{\alpha} \delta^{-\alpha} M^{-\alpha} \quad(n \rightarrow \infty) \\
& \rightarrow 0 \quad(M \rightarrow \infty)
\end{aligned}
$$

where in the next to last step we have used Proposition 4.2. For $B$ we have

$$
\begin{aligned}
& B \leq P\left[\bigcup_{t=1}^{m}\left\{\frac{\left\|\mathbf{X}_{t-1}(n)\right\|}{b(m) Z_{t}(n)}>\delta, \frac{\left\|\mathbf{X}_{t-1}(n)\right\|}{b(m)} \leq M^{-1}\right\}\right] \\
& \leq \sum_{t=1}^{m} P\left[\frac{\left\|\mathbf{X}_{t-1}(n)\right\|}{b(m) Z_{t}(n)}>\delta, Z_{t}(n) \leq(\delta M)^{-1}\right] \\
& \leq \sum_{t=1}^{m} P\left[\delta<\frac{\left\|\mathbf{X}_{t-1}(n)\right\|}{b(m)}\left\{\frac{1}{Z_{t}(n)} 1_{\left[\frac{1}{Z_{t}(n)}>M \delta\right]}\right\}\right] \\
& \leq m P\left[\delta<\sum_{j=0}^{\infty}\left\|\mathbf{c}_{j}(n)\right\| \frac{Z_{j}(n)}{b(m)}\left\{\frac{1}{Z^{\prime}(n)} 1_{\left[\frac{1}{Z^{\prime}(n)}>M \delta\right]}\right\}\right] \\
& =m P\left[\delta<X^{\prime}(n) \zeta_{M}(n)\right]
\end{aligned}
$$


where $\left\{Z^{\prime}(n), Z_{t}(n), t \geq 0\right\}$ are iid and

$$
\mathbf{X}^{\prime}(n)=b(m)^{-1} \sum_{j=0}^{\infty}\left\|\mathbf{c}_{j}(n)\right\| Z_{j}(n), \quad \zeta_{M}(n)=\frac{1}{Z^{\prime}(n)} 1_{\left[\frac{1}{Z^{\prime}(n)}>M \delta\right]} .
$$

From (4.18) in Proposition 4.2 we have

$$
\lim _{M \rightarrow \infty} \limsup _{n \rightarrow \infty} m P\left[\delta<X^{\prime}(n) \zeta_{M}(n)\right]=0
$$

and this finishes the proof.

\section{Validity of the bootstrap for linear programming estimators.}

The reader may wish to review the description of the bootstrap procedure given in steps (1) through (8) in Section 1. The theorem justifying this procedure is given next for the left tail case.

Theorem 5.1. Suppose conditions $M, S$ and $L$ hold so that the lp estimators have the limit distribution of the random vector $\mathbf{L}$ :

$$
a(n)^{-1}(\hat{\phi}(n)-\phi(0)) \Rightarrow \mathbf{L}
$$

Suppose $m=m(n) \rightarrow \infty$ in such a way that $m / n \rightarrow 0$ and that for $\beta$ appearing in condition $L$ we have $\beta>\alpha$ and that $m$ is further restricted to satisfy

$$
m\left(\frac{a(n)}{a(m)}\right)^{\beta} \rightarrow 0
$$

(This is the case, for instance, if $m / n^{\theta} \rightarrow 0$ for some $\theta<\beta /(\alpha+\beta)$.) Then

$$
P\left[a(m)^{-1}\left(\hat{\phi}^{*}(n)-\hat{\phi}(n)\right) \in \cdot \mid \hat{F}_{n}^{r e s i d}\right] \stackrel{P}{\rightarrow} P[\mathbf{L} \in \cdot]
$$

where the convergence in probability is in the space of random elements in $\mathcal{P} \mathcal{M}\left(\mathbb{R}^{p}\right)$, the probability measures on $\mathbb{R}^{p}$ topologized by weak convergence.

Proof. The subsequence characterization of convergence in probability means given any subsequence $\left\{n^{\prime \prime}\right\}$ of the positive integers, it suffices to show that there is a further subsequence $\left\{n^{\prime}\right\} \subset\left\{n^{\prime \prime}\right\}$ such that

$$
P\left[a\left(m\left(n^{\prime}\right)\right)^{-1}\left(\hat{\phi}^{*}\left(n^{\prime}\right)-\hat{\phi}\left(n^{\prime}\right)\right) \in \cdot \mid \hat{F}_{n}^{\text {resid }}\right] \stackrel{a . s .}{\rightarrow} P[\mathbf{L} \in \cdot] .
$$

The conditional distribution of $Z_{1}^{*}(n), \ldots, Z_{m}^{*}(n)$ is $\hat{F}_{n}^{\text {resid }}$ and this will play the role of $F_{n}$ in Theorem 4.1. So we need to know what analogues of (4.1)-(4.3) and (4.5) hold. These analogues are collected in the next lemma.

Lemma 5.2. Suppose the conditions of Theorem 5.1 are in force. Then we have the following properties of $\hat{F}_{n}^{r e s i d}$ :

(1) As $n \rightarrow \infty$

$$
\hat{F}_{n}^{\text {resid }} \stackrel{P}{\rightarrow} F
$$

in the sense of convergence in probability of random elements in the space $\mathcal{P} \mathcal{M}([0, \infty)$ of probability measures on $[0, \infty)$ topologized by weak convergence. 
(2) As $n \rightarrow \infty$

$$
m \hat{F}_{n}^{r e s i d}(a(m) \cdot) \stackrel{P}{\rightarrow} \nu
$$

in the sense of convergence in probability of random elements in $M_{+}[0, \infty)$ where $\nu[0, x)=x^{\alpha}, x>0$.

(3) Let $\left\{n_{k}\right\}$ be any sequence along which the lp estimators converge almost surely:

$$
\hat{\phi}\left(n_{k}\right) \stackrel{a . s .}{\rightarrow} \phi(0) .
$$

Then with probability one

$$
\sup _{n_{k}} \int_{0}^{\infty} t^{\beta} \hat{F}_{n_{k}}^{r e s i d}(d t)<\infty
$$

(4) $\operatorname{In} C_{0}[0, \infty)$, as $n \rightarrow \infty$

$$
m E\left(\left(\frac{Z_{1}^{*}(n)}{a(m)}\right)^{-\beta} 1_{\left[\left(\frac{Z_{1}^{*}(n)}{a(m)}\right)^{-1} \leq \eta\right]} \mid \hat{F}_{n}^{r e s i d}\right)=\int_{\eta^{-1}}^{\infty} x^{-\beta} m \hat{F}_{n}^{r e s i d}(a(m) d x) \stackrel{P}{\rightarrow} \frac{\alpha \eta^{\beta-\alpha}}{\beta-\alpha} .
$$

Proof. For (1) we let $g$ be positive, bounded, uniformly continuous on $[0, \infty)$ and set

$$
\omega_{g}(\delta)=\sup _{|x-y| \leq \delta}|g(x)-g(y)|, \quad\|g\|=\sup _{x} g(x) .
$$

Recall from $(2.14,2.15)$ that

$$
\Delta_{t}(n)=\hat{Z}_{t}(n)-Z_{t}=-(\hat{\phi}(n)-\phi(0))^{\prime} \mathbf{X}_{t-1},\left|\Delta_{t}(n)\right| \leq\|\phi(0)-\hat{\phi}(n)\|\left\|\mathbf{X}_{t-1}\right\|:=R_{t}(n) .
$$

For any $t$

$$
E\left|g\left(\hat{Z}_{t}(n)\right)-g\left(Z_{t}\right)\right|=E\left|g\left(Z_{t}+\Delta_{t}(n)\right)-g\left(Z_{t}\right)\right|
$$

and for any small $\delta$ and large $M$ this expectation is decomposed as

$$
\begin{aligned}
& =E\left(\left|g\left(Z_{t}+\Delta_{t}(n)\right)-g\left(Z_{t}\right)\right| ; R_{t}(n) \leq \delta\right)+E\left(\left|g\left(Z_{t}+\Delta_{t}(n)\right)-g\left(Z_{t}\right)\right| ; R_{t}(n)>\delta, \frac{\|\phi(0)-\hat{\phi}(n)\|}{a(n)}>M\right) \\
& \quad+E\left(\left|g\left(Z_{t}+\Delta_{t}(n)\right)-g\left(Z_{t}\right)\right| ; R_{t}(n)>\delta, \frac{\|\phi(0)-\hat{\phi}(n)\|}{a(n)} \leq M\right) \\
& =A+B+C .
\end{aligned}
$$

Now $A \leq \omega_{g}(\delta)$ and for $B$ we have as $n \rightarrow \infty$

$$
B \leq 2\|g\| P\left[\frac{\|\phi(0)-\hat{\phi}(n)\|}{a(n)}>M\right] \rightarrow 2\|g\| P[\|\mathbf{L}\|>M]
$$

which can be made arbitrarily small by choosing $M$ large. Finally for $C$ we get

$$
C \leq 2\|g\| P\left[R_{t}(n)>\delta, \frac{\|\phi(0)-\hat{\phi}(n)\|}{a(n)} \leq M\right] \leq 2\|g\| P\left[a(n) M\left\|\mathbf{X}_{t-i}\right\|>\delta\right] .
$$


The steps at the end of the proof of Proposition 3.2 show that this quantity goes to 0 as $n \rightarrow \infty$.

Now set

$$
\hat{F}_{n}=\frac{1}{n-p} \sum_{t=p+1}^{n} \epsilon_{Z_{t}}
$$

which converges to $F$. Then we have

$$
\begin{aligned}
\limsup _{n \rightarrow \infty} E\left|\hat{F}_{n}^{r e s i d}(g)-\hat{F}_{n}(g)\right| & \left.\leq \limsup _{n \rightarrow \infty} E\left[\frac{1}{n-p} \sum_{t=p+1}^{n} \mid g\left(\hat{Z}_{t}(n)\right)-g\left(Z_{t}\right)\right]\right] \\
& \leq \omega_{g}(\delta)+2\|g\| P[\|\mathbf{L}\|>M]+2\|g\| \limsup _{n \rightarrow \infty} P\left[a(n)\left\|\mathbf{X}_{t-1}\right\|>\delta / M\right] .
\end{aligned}
$$

The last term is zero and letting $\delta \downarrow 0$ and then $M \rightarrow \infty$ finishes the proof of (5.5).

(2) The proof of (5.6) is equivalent to (3.6) in Proposition 3.2.

(3) We have

$$
\int_{0}^{\infty} t^{\beta} \hat{F}_{n}^{\text {resid }}(d t)=\frac{1}{n-2 p} \sum_{t=p+1}^{n} \hat{Z}_{t}(n)^{\beta}=\frac{1}{n-2 p} \sum_{t=p+1}^{n}\left(Z_{t}+\Delta_{t}(n)\right)^{\beta}
$$

and we get an upper bound of

$$
\leq \frac{2^{\beta}}{n-2 p} \sum_{t=p+1}^{n} Z_{t}^{\beta}+\frac{2^{\beta}}{n-2 p} \sum_{t=p+1}^{n}\left|\Delta_{t}\right|^{\beta} .
$$

The first term on the right is bounded in $n$ by the strong law of large numbers since $E Z_{1}^{\beta}<\infty$. For the second term note

$$
\frac{2^{\beta}}{n-2 p} \sum_{t=p+1}^{n}\left|\Delta_{t}(n)\right|^{\beta} \leq\|\phi(0)-\hat{\phi}(n)\|^{\beta} \frac{2^{\beta}}{n-2 p} \sum_{t=p+1}^{n}\left(\left\|\mathbf{X}_{t-1}\right\|\right)^{\beta}
$$

Since

$$
\frac{1}{n-p} \sum_{t=p+1}^{n}\left(\left\|\mathbf{X}_{t-1}\right\|\right)^{\beta} \stackrel{\text { a.s. }}{\rightarrow} E\left(\left\|\mathbf{X}_{1}\right\|\right)^{\beta}<\infty
$$

and

$$
\|\phi(0)-\hat{\phi}(n)\| \stackrel{a . s .}{\rightarrow} 0
$$

along the hypothesized subsequence $\left\{n_{k}\right\}$ the assertion (5.7) is proven.

(4) We know from (3.7) that in $C_{0}[0, \infty)$

$$
\int_{0}^{x} \hat{F}_{n}^{r e s i d}\left(a(m) u^{-1}\right) u^{\beta-1} d u \stackrel{P}{\rightarrow} \frac{x^{\beta-\alpha}}{\beta-\alpha}
$$

and we use this plus integration by parts to get (5.8). To do this set

$$
c(m)=1 / a(m), \quad 1-\hat{G}_{n}(c(m) u)=\hat{F}_{n}^{r e s i d}\left(\frac{1}{c(m) u}\right) .
$$

Now

$$
\int_{0}^{x} m\left(1-\hat{G}_{n}(c(m) u) \beta u^{\beta-1} d u-m\left(1-\hat{G}_{n}(c(m) x)\right) x^{\beta}=\int_{0}^{x} u^{\beta} m \hat{G}_{n}(c(m) d u) .\right.
$$


Since

$$
m\left(1-\hat{G}_{n}(c(m) x)\right) x^{\beta}=m \hat{F}_{n}^{r e s i d}\left(a(m) x^{-1}\right) x^{\beta} \stackrel{P}{\rightarrow} x^{\beta-\alpha},
$$

we have

$$
\int_{0}^{x} u^{\beta} m \hat{G}_{n}(c(m) d u) \stackrel{P}{\rightarrow} \frac{\beta}{\beta-\alpha} x^{\beta-\alpha}-x^{\beta-\alpha}=\frac{\alpha x^{\beta-\alpha}}{\beta-\alpha} .
$$

The proof is finished by noting that

$$
\int_{0}^{x} u^{\beta} m \hat{G}_{n}(c(m) d u)=\int_{x^{-1}}^{\infty} s^{-\beta} \hat{F}_{n}^{r e s i d}(a(m) d s) .
$$

Continuation of the proof of Theorem 5.1. Remember that our goal is that given any subsequence $\left\{n^{\prime \prime}\right\}$ we need a further subsequence $\left\{n^{\prime}\right\} \subset\left\{n^{\prime \prime}\right\}$ such that (5.3) holds. From (5.5)-(5.8) and the fact that $\phi(n) \stackrel{P}{\rightarrow} \phi(0)$, a subsequence $\left\{n^{\prime}\right\}$ exists such that along this subsequence the almost sure analogues of (5.5), (5.6), (5.8) (do not overlook that $(5.8)$ is in $C[0, \infty)$ ) hold as well as (5.7). Now apply Theorem (4.1) to get almost surely along this subsequence that

$$
P\left[\sum_{i=1}^{m} \epsilon_{\left(a(m) Z_{t}^{*}(n)^{-1} \mathbf{X}_{t-j}^{*}(n)\right)} \in \cdot \mid \hat{F}_{n}^{r e s i d}\right] \rightarrow P\left[\sum_{k} \epsilon_{\left(j_{k}^{-1} \mathbf{Y}_{k}\right)} \in \cdot\right]
$$

in $\mathcal{P} \mathcal{M}\left(M_{p}\left(([-\infty, \infty] \backslash\{0\})^{p}\right)\right)$. Applying Theorems 5.1, 6.1, 3.5 and 3.8 in Feigin and Resnick (1994) yields the desired conclusion 5.3.

Theorem 5.1 has the defect that it is expressed in terms of the unknown quantity $a(m)$; if $F$, the distribution of $\left\{Z_{n}\right\}$, is unknown then so is the quantile function of $F$. There are several ways to rectify this and the following is a simple solution which uses the known estimated residuals. The idea is that the empirical distribution of the estimated residuals is close to $F$ (see (5.6) and Theorem 6.1 in Feigin, Resnick and Starica (1994)) and therefore the quantile of this empirical should be close to the quantile of $F$.

Let

$$
0<\hat{Z}_{(n, 1)}(n) \leq \hat{Z}_{(n, 2)}(n) \leq \cdots \leq \hat{Z}_{(n, n-2 p)}(n)
$$

be the order statistics of the estimated non-zero residuals from $\hat{Z}_{1}(n), \ldots, \hat{Z}_{n-p}(n)$ arranged in increasing order. Then from (5.6) and Proposition 6.2 of Feigin, Resnick and Starica (1994) (see Starica and Resnick (1995) for a discussion of the basic method) we get

$$
\hat{Z}_{\left(n,\left[\frac{n}{m}\right]\right)}(n) / a(m) \stackrel{P}{\rightarrow} 1,
$$

as $n \rightarrow \infty$. This leads to the following modification of Theorem 5.1.

Corollary 5.3. Suppose the conditions of Theorem 5.1 hold. Then

$$
P\left[\hat{Z}_{\left(n,\left[\frac{n}{m}\right]\right)}(n)^{-1}\left(\hat{\phi}^{*}(n)-\hat{\phi}(n)\right) \in \cdot \mid \hat{F}_{n}^{r e s i d}\right] \stackrel{P}{\rightarrow} P[\mathbf{L} \in \cdot]
$$

where the convergence in probability is in the space of random elements in $\mathcal{P} \mathcal{M}\left(\mathbb{R}^{p}\right)$, the probability measures on $\mathbb{R}^{p}$ topologized by weak convergence.

Proof. We claim that

$$
P\left[a(m)^{-1}(\hat{\phi}(n)-\phi(0)) \in \cdot, \frac{\hat{Z}_{\left(n,\left[\frac{n}{m}\right]\right)}(n)}{a(m)} \in \cdot \mid \hat{F}_{n}^{r e s i d}\right] \stackrel{P}{\rightarrow} P[\mathbf{L} \in \cdot] \times \epsilon_{1}(\cdot) .
$$


To see this, note for any $\delta$ and set $B \in \mathcal{B}\left(\mathbb{R}^{p}\right)$ that

$$
P\left[a(m)^{-1}(\hat{\phi}(n)-\phi(0)) \in B,\left|\frac{\hat{Z}_{\left(n,\left[\frac{n}{m}\right]\right)}(n)}{a(m)}-1\right|>\delta \mid \hat{F}_{n}^{\text {resid }}\right] \stackrel{P}{\rightarrow} 0
$$

as $n \rightarrow \infty$ since the expectation is bounded above by

$$
P\left[\left|\frac{\hat{Z}_{\left(n,\left[\frac{n}{m}\right]\right)}(n)}{a(m)}-1\right|>\delta\right] \rightarrow 0 .
$$

Also

$$
\begin{aligned}
& P\left[a(m)^{-1}(\hat{\phi}(n)-\phi(0)) \in B,\left|\frac{\hat{Z}_{\left(n,\left[\frac{n}{m}\right]\right)}(n)}{a(m)}-1\right| \leq \delta \mid \hat{F}_{n}^{r e s i d}\right] \\
& =1\left[\mid \frac{\left.\hat{z}_{\left(n,\left[\frac{n}{m}\right]\right)^{(n)}}^{a(m)}-1 \mid \leq \delta\right]}{} P\left[a(m)^{-1}(\hat{\phi}(n)-\phi(0)) \in B \mid \hat{F}_{n}^{\text {resid }}\right]\right. \\
& \stackrel{P}{\rightarrow} 1 P[\mathbf{L} \in B] .
\end{aligned}
$$

By dividing the two components of (5.11) we get (5.10).

Now we give the results for the right tail case.

Proposition 5.4. Suppose conditions $M, S$ and $R$ hold so the lp estimators have limit distribution $\mathbf{L}$ described in Theorem 1.1:

$$
b(n)(\hat{\phi}(n)-\phi(0)) \Rightarrow \mathbf{L} .
$$

Suppose $m \rightarrow \infty$ and $m / n \rightarrow 0$. Then

$$
P\left[b(m)\left(\hat{\phi}^{*}(n)-\hat{\phi}(n)\right) \in \cdot \mid \hat{F}_{n}^{r e s i d}\right] \stackrel{P}{\rightarrow} P[\mathbf{L} \in \cdot] .
$$

Proof. Given $\left\{n^{\prime \prime}\right\}$, we need to find a subsequence $\left\{n^{\prime}\right\} \subset\left\{n^{\prime \prime}\right\}$ such that

$$
P\left[b\left(m\left(n^{\prime}\right)\right)\left(\hat{\boldsymbol{\phi}}^{*}\left(n^{\prime}\right)-\hat{\boldsymbol{\phi}}\left(n^{\prime}\right)\right) \in \cdot \mid \hat{F}_{n}^{\text {resid }}\right] \rightarrow P[\mathbf{L} \in \cdot],
$$

almost surely. As before, the conditional distribution of $Z_{1}^{*}(n), \ldots, Z_{m}^{*}(n)$ is $\hat{F}_{n}^{\text {resid }}$ and this plays the role of $F_{n}$ in Theorem 4.3. Pick a subsequence $\left\{n^{\prime}\right\}$ such that on $\left\{n^{\prime}\right\}$ we have $\hat{\phi}\left(n^{\prime}\right) \rightarrow \phi(0)$ a.s. and (3) and (4) of Proposition 3.3 hold a.s. which empowers Theorem 4.2. Thus, to get the subsequential analogue of (4.28) of Theorem 4.3 where $\hat{F}_{n}^{\text {resid }}$ replaces $F_{n}$ to hold on $\left\{n^{\prime}\right\}$, we need to check

$$
\sup _{n^{\prime} \geq 1} \int_{0}^{M^{-1}} x^{-\beta} \hat{F}_{n^{\prime}}^{r e s i d}(d x)<\infty
$$

almost surely. If we can prove this fact, the remainder of the proof of Proposition 5.4 follows similar lines to that of the left tail case and will be omitted.

To prove (5.12) it suffices to show we can construct an infinite sequence $\Lambda^{*}$ of positive integers such that

$$
\sup _{n \in \Lambda^{*}} \frac{1}{n} \sum_{t=1}^{n} \hat{Z}_{t}(n)^{-\beta} 1_{\left[\hat{Z}_{t}(n)>0\right]}<\infty
$$


almost surely. Using the notation in (2.11-2.13), and setting $M=\frac{1}{2 \eta}$, we note

$$
\begin{aligned}
\frac{1}{n} \sum_{t=1}^{n} \hat{Z}_{t}(n)^{-\beta} 1_{\left[\hat{Z}_{t}(n)>0\right]}= & \frac{1}{n} \sum_{t=1}^{n} \hat{Z}_{t}(n)^{-\beta} 1_{\left[\hat{Z}_{t}(n)>0,\left\|\mathbf{u}_{t}(n)\right\|>\eta\right]} \\
& +\frac{1}{n} \sum_{t=1}^{n} Z_{t}(n)^{-\beta}\left(1-\hat{\boldsymbol{\delta}}(n)^{\prime} \mathbf{u}_{t}(n)\right)^{-\beta} 1_{\left[\hat{Z}_{t}(n)>0,\left\|\mathbf{u}_{t}(n)\right\| \leq \eta,\|\hat{\boldsymbol{\delta}}(n)\| \leq M\right]} \\
& +\frac{1}{n} \sum_{t=1}^{n} Z_{t}(n)^{-\beta}\left(1-\hat{\boldsymbol{\delta}}(n)^{\prime} \mathbf{u}_{t}(n)\right)^{-\beta} 1_{\left[\hat{Z}_{t}(n)>0,\left\|\mathbf{u}_{t}(n)\right\| \leq \eta,\|\hat{\boldsymbol{\delta}}(n)\| \geq M\right]} \\
= & A_{n}(M)+B_{n}(M)+C_{n}(M) .
\end{aligned}
$$

We first consider $A_{n}(M)$ - note that $A_{n}(M)$ depends on $M$ since $\eta$ is a function of $M$. Start by observing that

$$
\left(\sum_{t=1}^{n} \epsilon_{\left(Z_{t}, \mathbf{u}_{t}(n)\right)}, \hat{\boldsymbol{\delta}}(n)\right) \Rightarrow\left(\sum_{k, l} \epsilon_{\left(Y_{k, l}, Y_{k, l}^{-1} j_{k} \mathbf{c}_{l}\right)}, \mathbf{L}\right)
$$

in $M_{p}\left((0, \infty) \times[-\infty, \infty]^{p} \backslash\{0\}\right) \times \mathbb{R}$. This follows because $\hat{\boldsymbol{\delta}}(n)$ is a function of the first component in the convergence. Thus for $\eta=\frac{1}{2 M}>0$

$$
\left(\sum_{t=1}^{n} 1_{\left[\left\|\mathbf{u}_{t}(n)\right\|>\eta, \hat{Z}_{t}(n)>0\right]} \epsilon_{\left(Z_{t}, \mathbf{u}_{t}(n)\right)}, \hat{\boldsymbol{\delta}}(n)\right) \Rightarrow\left(\sum_{k, l} 1_{\left[\left\|Y_{k, l}^{-1} j_{k} \mathbf{c}_{l}\right\|>\eta, 1-Y_{k, l}^{-1} j_{k} \mathbf{L}^{\prime} \mathbf{c}_{l}>0\right]} \epsilon_{\left(Y_{k, l}, Y_{k, l}^{-1} j_{k} \mathbf{c}_{l}\right)}, \mathbf{L}\right)
$$

in $M_{p}((0, \infty) \times\{\mathbf{x}:|\mathbf{x}|>\eta\}) \times \mathbb{R}$. So

$$
\sum_{t=1}^{n} 1_{\left[\left\|\mathbf{u}_{t}(n)\right\|>\eta, \hat{Z}_{t}(n)>0\right]^{\epsilon}} \epsilon_{\left.t, 1-\hat{\delta}(n)^{\prime} \mathbf{u}_{t}(n)\right)} \Rightarrow\left(\sum_{k, l} 1_{\left[\left\|Y_{k, l}^{-1} j_{k} \mathbf{c}_{l}\right\|>\eta, 1-Y_{k, l}^{-1} j_{k} \mathbf{L}^{\prime} \mathbf{c}_{l}>0\right]} \epsilon_{\left(Y_{k, l}, 1-Y_{k, l}^{-1} j_{k} \mathbf{c}_{l}^{\prime} \mathbf{L}\right)}\right.
$$

where the limit has a finite number of terms. Thus we get

$$
\frac{1}{n} \sum_{t=1}^{n} 1_{\left[\left\|\mathbf{u}_{t}(n)\right\|>\eta, \hat{Z}_{t}(n)>0\right]} Z_{t}^{-\beta}\left(1-\hat{\boldsymbol{\delta}}(n)^{\prime} \mathbf{u}_{t}(n)\right)^{-\beta} \stackrel{P}{\rightarrow} 0 .
$$

Thus for fixed $M, A=o_{p}(1)$ as $n \rightarrow \infty$.

For $B_{n}(M)$, with $M \eta=1 / 2$,

$$
B_{n}(M) \leq\left(\frac{1}{2}\right)^{-\beta} \frac{1}{n} \sum_{t=1}^{n} Z_{t}^{-\beta} \equiv B_{n}^{*}
$$

which is bounded almost surely by the assumption that $E Z_{1}^{-\beta}<\infty$.

For $C_{n}(M)$, note that

$$
P\left[C_{n}(M)>0\right] \leq P[\|\hat{\delta}(n)\|>M] .
$$

Since $\{\hat{\boldsymbol{\delta}}(n)\}$ is convergent and hence tight, given $\epsilon>0$, we can choose $M$ so large that

$$
P[\|\hat{\delta}(n)\|>M] \leq \epsilon,
$$

for all $n$. Thus, for every $l=1,2, \ldots$, there exists $M_{l}$ such that for all $n$

$$
P\left[\|\hat{\delta}(n)\|>M_{l}\right]<\frac{1}{2^{l}} .
$$


So for each fixed $l$, there exist an infinite family of positive integers

$$
\Lambda_{l}=\left\{n_{k}\left(M_{l}\right), k \geq 1\right\}
$$

such that

$$
\Lambda_{l+1} \subset \Lambda_{l}, \quad l=1,2, \ldots
$$

and

$$
P\left[\sup _{n \in \Lambda_{l}} A_{n}\left(M_{l}\right)+B_{n}^{*}<\infty\right]=1
$$

and therefore

$$
P\left[\sup _{n \in \Lambda_{l}} A_{n}\left(M_{l}\right)+B_{n}\left(M_{l}\right)<\infty\right]=1
$$

Now define

$$
\Lambda^{*}=\left\{n_{l}\left(M_{l}\right), l \geq 1\right\}
$$

so that $\Lambda^{*} \subset \Lambda_{l}$ for any $l$. Also,

$$
\sum_{l} P\left[C_{n_{l}\left(M_{l}\right)}\left(M_{l}\right)>0\right]<\sum_{l} \frac{1}{2^{l}}<\infty
$$

so by Borel-Cantelli

$$
P\left[C_{n_{l}\left(M_{l}\right)}\left(M_{l}\right)>0 \text { i.o. }\right]=0
$$

and with probability one, for all sufficiently large $l, C_{n_{l}\left(M_{l}\right)}\left(M_{l}\right)=0$.

Thus we have

$$
\begin{aligned}
P\left[\sup _{n \in \Lambda^{*}}\right. & \left.\frac{1}{n} \sum_{t=1}^{n} \hat{Z}_{t}(n)^{-\beta} 1_{\left[\hat{Z}_{t}(n)>0\right]}<\infty\right] \\
\quad & P\left[\sup _{l}\left(A_{n_{l}\left(M_{l}\right)}\left(M_{l}\right)+B_{n_{l}\left(M_{l}\right)}\left(M_{l}\right)\right)<\infty\right] \\
& \geq P\left[\sup _{n \in \Lambda_{l}}\left(A_{n}\left(M_{l}\right)+B_{n}\left(M_{l}\right)\right)<\infty\right]=1
\end{aligned}
$$

for any $l$. This completes the proof since $\Lambda^{*}$ supplies the needed subsequence.

Dependence on the unknown $b(m)$ can be removed as in the left tail case.

\section{REFERENCES}

Athreya, K., Bootstrap of the mean in the infinite variance case, Ann. Statist. 15 (1987), 724-731.

Billingsley, P., Convergence of Probability Measures, Wiley, New York, 1968.

Bingham,N., Goldie, C. and Teugels, J., Regular Variation, Encyclopedia of Mathematics and its Applications, Cambridge University Press, Cambridge, UK, 1987.

Cline, D., Estimation and linear prediction for regression, autoregression and ARMA with infinite variance data., Thesis, Dept of Statistics, Colorado State University, Ft. Collins CO 80521 USA.

Datta, S. and McCormick, W., Bootstrap inference for a first order autoregression with positive innovations, To appear December 1995, JASA (1995).

Davis, R. and McCormick, W., Estimation for first order autoregressive processes with positive or bounded innovations, Stochastic Processes and their Appl. 31 (1989), 237-250.

Davis, R. and Resnick, S., Limit theory for moving averages of random variables with regularly varying tail probabilities., Ann. Probability 13 (1985), 179-195.

Davis, R. and Resnick, S., Extremes of moving averages of random variables from the domain of attraction of the double exponential distribution, Stochastic Processes and their Appl. 30 (1988), 41-68. 
Deheuvels, P., Mason, D. and Shorack, G., Some results on the influence of extremes on the bootstrap, Ann. Inst. Henri Poincare 29 (1993), 83-103.

Feigin, P. and Resnick, S., Estimation for autoregressive processes with positive innovations, Stochastic Models 8 (1992), 479-498.

Feigin, P. and Resnick, S., Limit distributions for limear programming time series estimators, Stochastic Processes and their Applications 51 (1994), 135-166.

Feigin, P., Resnick, S. and Starica, C., Testing for independence in heavy tailed and positive innovation time series, Submitted (1994).

Feller, W., An Introduction to Probability Theory and its Applications, Vol. II, second edition, Wiley, New York, 1971.

Geluk, J. and Haan, L. de, Regular Variation, Extensions and Tauberian Theorems, CWI Tract 40, Center for Mathematics and Computer Science, P.O. Box 4079, 1009 AB Amsterdam, The Netherlands, 1987.

Gine, E. and Zinn, J., Necessary conditions for the bootstrap of the mean, Ann. Statist. 17 (1989), 684-691.

Haan, L. de, On Regular Variation and its Application to the Weak Convergence of Sample Extremes, Mathematical Centre Tract 32, Mathematical Centre, Amsterdam, Holland, 1970.

Hall, P., Asymptotic properties of the bootstrap for heavy-tailed distributions, Ann. Probability 18 (1990), 1342-1360.

Kallenberg, O., Random Measures, Third edition, Akademie-Verlag, Berlin, 1983.

Kinateder, J., An invariance principle applicable to the bootstrap, Exploring the Limits of Boostrap (R. LePage and Lynne Billard, ed.), John Wiley and Sons, New York, 1992.

Knight, K., On the bootstrap of the sample mean in the infinite variance case, Ann. Statist. 17 (1989), $1168-1175$.

LePage, R., Bootstrapping signs, Exploring the Limits of Boostrap (R. LePage and Lynne Billard, ed.), John Wiley and Sons, New York, 1992.

Resnick, Sidney, Point processes, regular variation and weak convergence, Advances Appl. Probability 18 (1986), 66-138.

Resnick, Sidney, Extreme Values, Regular Variation, and Point Processes, Springer-Verlag, New York, 1987.

Resnick, S. and Starica, C., Consistency of Hill's estimator for dependent data, Preprint: School of ORIE, Cornell University, ETC Building, Ithaca, NY 14853, USA, To appear: J. Appl. Probability (1995).

Rudin, W., Real and Complex Analysis, McGraw Hill Book Company, New York, 1966.

Paul D. Feigin, Faculty of Industrial Engineering and Management, TECHniOn - Israel Institute of TechNOLOGY, HAIFA 32000 , ISRAEL

E-mail: paulf@ie.technion.ac.il

Sidney I. Resnick, Cornell University, School of Operations Research and Industrial EngineERing, ETC BuildING, ITHACA, NY 14853 USA

E-mail: sid@orie.cornell.edu 